Article

\title{
Genome-Wide Identification of WRKY Transcription Factors in Chinese jujube (Ziziphus jujuba Mill.) and Their Involvement in Fruit Developing, Ripening, and Abiotic Stress
}

\author{
Xin Chen ${ }^{1,2}$, Ruihong Chen ${ }^{3}$, Yanfeng Wang ${ }^{3}$, Cuiyun $\mathrm{Wu}^{2}$ and Jian Huang ${ }^{1,4, *}$ \\ 1 Key Comprehensive Laboratory of Forest for Shaanxi Province, College of Forestry, Northwest A\&F \\ University, Yangling 712100, China; chenxin0526@126.com \\ 2 Xinjiang Production \& Construction Corps Key Laboratory of Protection and Utilization of Biological \\ Resources in Tarim Basin, College of Plant Science, Tarim University, Alaer 843300, China; wcyby@163.com \\ 3 Shaanxi Key Laboratory of Chinese Jujube, College of Life Science, Yanan University, Yanan 716000, China; \\ chenruihong328@163.com (R.C.); yadxwyf@yau.edu.cn (Y.W.) \\ 4 Institute of Loess Plateau, Shanxi University, Taiyuan 030006, China \\ * Correspondence: huangj@nwsuaf.edu.cn; Tel.: +86-1809-202-6218
}

Received: 25 February 2019; Accepted: 6 May 2019; Published: 10 May 2019

\begin{abstract}
Chinese jujube (Ziziphus jujuba Mill.) is an economically important fruit crop in China and mainly cultivated on land with high salinity and drought conditions in northern China. WRKY transcription factors (TFs) are involved in plant development and in responses to multiple abiotic stresses. In this study, we identified 61 and 52 putative ZjWRKY TFs in 'Junzao' and 'Dongzao' at the genome-wide level. Tissue expression profiling showed that 7 genes were constitutively expressed at high level in all tissues of 'Junzao'. Transcriptome analysis revealed that 39 ZjWRKY genes were expressed during 'Junzao' jujube fruit ripening. Among these genes, the transcript abundance of 19 genes were differentially expressed between 'Junzao' and 'Qingiiansuanzao' fruit. In addition, RT-qPCR analyses revealed that 30,14, and 18 ZjWRKY genes responded to drought, $\mathrm{NaCl}$, and ABA treatments, respectively. Taken together, ZjWRKY genes expression dynamics during jujube fruit development, ripening, and their differences between jujube and wild jujube would provide insights into their possible roles regulating fruit ripening. In addition, those ZjWRKY genes responded strongly to drought and salt stress, which provide candidate ZjWRKY genes for facilitating tolerance breeding.
\end{abstract}

Keywords: Ziziphus jujuba Mill.; WRKY; transcription factors; fruit ripening; drought stress; salt stress

\section{Introduction}

Chinese jujube (Ziziphus jujuba Mill.) is a dominant fruit crops in China that is mainly cultivated in the middle and lower reaches of the Yellow River, a semi-arid region. Since the beginning of the 21st century, the center of jujube cultivation has shifted to arid regions in Northwest China, especially the Xinjiang Autonomous Region [1]. In this region, the jujube cultivation area covered 473,000 ha, accounting for $30 \%$ of the total jujube cultivation area in China in 2013, and the corresponding yield was 2.22 million tons, accounting for $51 \%$ of the total production of dried jujube in China [2]. In the Xinjiang jujube cultivation area, there is a long sunshine duration $(>1200 \mathrm{~h})$, a large temperature difference between day and night $\left(>12^{\circ} \mathrm{C}\right)$, and low rainfall $(0.2-9.38 \mathrm{~mm})$ during jujube fruit ripening, all of which might contribute to the jujube fruit quality [3]. For example, the sugar content (73.2\%) of 'Huizao'

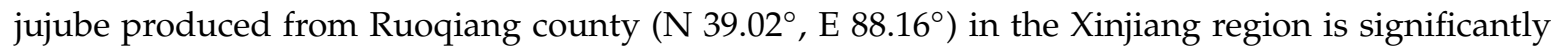

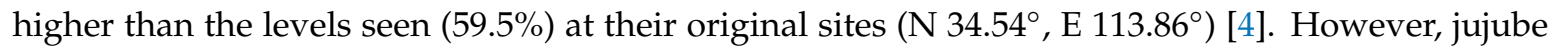


trees are also constantly exposed to extremely adverse conditions in this region, such as soil salinity, drought, and very high or low temperatures. Thus, the jujube tree is likely to have evolved a series of adaptation strategies to cope with such unfavorable conditions [5].

Most abiotic stress, such as high salinity and drought, disrupt the osmotic pressure in plants [6]. In addition, high salt concentrations can lead to ionic toxicity and secondary stress. The $\mathrm{Ca}^{2+}$ secondary signal caused by stress could activate related transcription factors (TFs) through the abscisic acid-dependent (ABA-dependent) or mitogen-activated protein kinase pathways [7]. Thereafter, TFs can activate the transcription of specific genes to regulate the physiological and biochemical responses to stress. Thus, TFs play an essential role in the complex regulatory networks of plants. As one of the largest families of TFs in plants, WRKY TFs are involved in regulating plant tolerance to biotic and abiotic stresses, and in plant development [8]. Almost all WRKY proteins contain one or two conserved domains of approximately 60 amino acids containing a conserved heptapeptide WRKYGQK followed by a $\mathrm{C}_{2} \mathrm{H}_{2}$ or $\mathrm{C}_{2} \mathrm{HC}$ zinc finger structure [9]. The WRKY proteins activate or inhibit the expression of target genes by recognizing and binding to a W-box (C/TTGACT/C) in the promoter region of target genes. WRKY TFs are usually divided into three groups, depending on the number of WRKY domains and the type of zinc finger structure. In some studies, WRKY TFs with incomplete zinc finger structures have been assigned to group IV [10].

Several studies have confirmed the function of WRKY TFs regulating plant responses to abiotic stresses. Several WRKY genes, such as AtWRKY33, 46, 57 enhance Arabidopsis thaliana tolerance to drought/salinity by mediating ABA signal transduction [11-13]. In recent years, WRKY TFs identification at the genome-wide level have been facilitated by the greater availability of the genome sequences of fruit crops, such as Malus domestica [14], Citrus sinensis [15], and Vitis vinifera [10]. The functions of some WRKY genes have also been further verified in some species. In Fortunella crassifolia, FcWRKY40 enhances salt tolerance by regulating ion homeostasis and proline synthesis dependent on the ABA signal transduction pathway [16]. VvWRKY30 positively regulates drought response by modulating proline and soluble sugar metabolism, as well as activating reactive oxygen scavenging systems [17]. Considering the vital role of WRKY TFs response to multiple stresses, it would be valuable to characterize the WRKY TFs in the jujube genome.

In addition to responding to abiotic stresses, WRKY TFs are also involved in a variety of biological processes such as leaf senescence [18], trichome, seed and pollen development [19,20], and secondary metabolite biosynthesis [21]. Recently, some studies have revealed that WRKY TFs may be involved in fruit development and ripening [22,23]. Jujube was domesticated from its wild ancestor (Z. jujuba Mill. var. spinosa $\mathrm{Hu}$.), which resulted in enlarged fruit sizes and changes in fruit taste such as increased sweetness/acidity. In our previous report, we have elucidated the difference in fruit taste between the wild jujube and jujube fruit through transcriptomic analysis and genomic selection [24]. However, there were still lack of detail information about TFs involved in jujube fruit development and ripening. Therefore, it is valuable to study the expression pattern of WRKY genes, one of the largest plant TF families, and their differential expression between jujube and wild jujube during fruit ripening, and hope to reveal the potential role of WRKY genes involved in fruit ripening.

In this study, we identified and compared WRKY TFs in two genome-sequenced jujube cultivars, i.e., 'Junzao' and 'Dongzao', at the genome-wide level, and characterized their chromosomal location and phylogenetic relationships. 'Junzao' is a dominant dry-eating jujube cultivar with large size and high sugar content, while 'Dongzao' is the most dominant fresh-eating cultivar with crisp flesh and good tastes in China. We analyzed the expression profiles of WRKY TFs in different tissues of 'Junzao'. To elucidate their potential role in regulating fruit development and ripening, we compared the transcription patterns of cultivated and wild jujube during fruit ripening. Finally, we performed pot and hydronic experiments and studied the responses of different $Z j W R K Y$ genes to drought stress, salt stress, and ABA treatment using RT-qPCR. The results would help us understand the molecular mechanisms of jujube adaptation to harsh conditions and provide gene resources for jujube molecular breeding to improve stress tolerance and fruit quality. 


\section{Materials and Methods}

\subsection{Identification of the WRKY Family Members in Chinese Jujube and Their Chromosomal Locations}

The protein-coding sequences and their corresponding chromosomal locations in the jujube genome were downloaded from our previously released data of 'Junzao' jujube genome (https: //datadryad.org/resource/doi:10.5061/dryad.83fr7). The Hidden Markov Model profiles for the WRKY DNA-binding domain (PF03106) were retrieved from the Pfam data base (http://pfam.xfam.org/) [25] and used to identify jujube WRKY genes (E-Value < 0.01) with HMMER 3.0 (http://hmmer.janelia.org/) [26]. Pfam domains and NCBI CDD (http://www.ncbi.nlm.nih.gov/cdd/) were used to validate all the candidate WRKY TFs. We also identified WRKY gene family in 'Dongzao' jujube genome (https: //www.ncbi.nlm.nih.gov/genome/?term=jujube). We compared amino acids composition of WRKY TFs between 'Junzao' and 'Dongzao' using BlastP.

\subsection{Sequence Alignment of WRKY Family Members and Construction of the Phylogenetic Tree}

Conserved domain amino acid (aa) sequences of all predicted ZjWRKY were multi-aligned using ClustalX 2.0.12 [27]. Another multi-alignment, including aa sequences of jujube and those from $A$. thaliana and Solanum lycopersicum, was performed using ClustalW and MEGA 7.0 with the neighbor-joining and maximum likelihood method (bootstrap $=1000$ ) [28]. Based on the results of multiple sequence alignment and the classification of $A$. thaliana WRKY TFs, all predicted ZjWRKY genes were assigned to groups and subgroups.

\subsection{Structural and Analysis of WRKY Genes}

The online ExPASy proteomics server (https://web.expasy.org/protparam/) was used to predict protein molecular weights (MWs) and isoelectric points (pIs) of the putative WRKY proteins. Motifs in all the predicted ZjWRKY protein sequences were identified using the Multiple Em for Motif Elicitation (MEME) 5.0.1 online program (http://meme-suite.org/tools/meme) [29]. The parameters for MEME were as follows: number of repetitions, any; maximum number of motifs, 10; and the optimum width of each motif, between 20 and 50 residues. To evaluate the level of conservation of the WRKY structural domains and zinc finger motifs of each group, sequence logos were produced using Jalview 2.10.4b1.

Both gene sequence and coding sequence (CDS) of each predicted ZjWRKY gene from the Z. jujuba genome were downloaded, and the intron distribution pattern and splicing phase were analyzed using the gene structure display server (GSDS) (http://gsds.cbi.pku.edu.cn/) [30].

\subsection{Analysis of Cis-Acting Regulatory Elements in the Promoter Regions of ZjWRKY Genes}

We extracted the region of $2000 \mathrm{bp}$ upstream of the transcriptional start point of ZjWRKY genes from 'Junzao' and 'Dongzao' jujube genome [22], and identified cis-acting regulatory elements in these regions using PlantCARE [31].

\subsection{Tissue-Specific Expression Analysis of ZjWRKY Genes by Reverse Transcription Polymerase Chain Reaction (RT-PCR)}

We performed RT-PCR analysis to study the ZjWRKY genes expression in different tissues, including roots, stems, flowers, and leaves of 'Junzao'. We collected at least $30 \mathrm{~g}$ leaves, stems and fine roots, 30 young fruits, 10 ripe fruits, and $3 \mathrm{~g}$ flower samples from one tree per replicate, respectively. Three biological replicates for each type of tissues were collected totally. These samples were harvested on June 16, 2017, from three 10-year-old 'Junzao' jujube trees growing in the Jujube Experimental Station of Northwest A\&F University (Qingjian, Shaanxi, China; N 37.13, E $110.09^{\circ}$ ). Over 50\% flowers were fully blooming on June 16, 2017 in the experimental station and this time was defined as 0 days after flowering (DAF), young fruits were collected at 15 DAF (1 July 2017) and ripe fruits were collected at 110 DAF (4 October 2017). In addition, we dissected flowers into 4 parts, included sepals, disks, 
pistils, and stamen. All prepared samples were frozen in liquid nitrogen and then transferred to a $-80^{\circ} \mathrm{C}$ freezer for storage.

Total RNA was extracted using a Plant RNA Extraction Kit (Takara, Dalian, China) and DNA was removed using DNase I. The RNA was quantified using a NanoDrop 2000 UV-vis spectrophotometer (Thermo Fisher Scientific, USA), and its integrity was confirmed using 1\% agarose gel electrophoresis. cDNA was synthesized by the PrimeScript ${ }^{\mathrm{TM}} \mathrm{RT}$ reagent Kit (Takara, Dalian, China). Primer sets for 61 ZjWRKY genes identified from 'Junzao' jujube were designed by NCBI Primer-BLAST (https://www. ncbi.nlm.nih.gov/tools/primer-blast/). The parameters for Primer-BLAST were as follows: PCR product size, 80 180bp; melting temperatures (Tm), 57 60; the forward/reverse primer range was specified to span one intron; forward/reverse primer length: 19 22 bp, GC\%: 50 55, self complementarity: 0 6, self 3' complementarity: 0 3. ZjUBQ (GenBank Accession: EU916200.1; Forward primer: 5'-TGGATGATTCTGGCAAAG-3'; Reverse primer: 5'-GTAATGGCGGTCAAAGTG-3') was selected as reference for RT-PCR analysis [32]. RT-PCR was performed using $2 \times$ GoldStar BesterMix (CWBIO, Beijing, China) on an ABI 2720 Thermal Cycler (ABI, Marsiling, Singapore). PCR products were detected by $1.2 \%$ agarose electrophoresis. Three technical replicates were performed for each sample. The band intensity was detected using Image J [33].

\subsection{Transcriptome Analysis of ZjWRKY Genes During Jujube and Wild Jujube Fruit Development and Ripening}

Wild jujube is the wild ancestors of jujube, which have small fruit size and acidity taste in contrast cultivated jujubes with large fruit size and sweet taste [24]. To identify the WRKY TFs that may be associated with jujube fruit size enlargement or sugar accumulation, we compared their expression level of WRKY TFs between wild and cultivated jujube fruits based on transcriptomic analysis. We harvested cultivated jujube ('Junzao') and wild jujube ('Qingjiansuanzao') fruits at five stages of fruit ripening: young (15 DAF), enlarging (40 DAF), white mature (65 DAF), beginning red (90 DAF), and fully red (110 DAF) from 10-year-old trees, respectively, which were grown in Jujube Experimental Station of Northwest A\&F University under the same environment and management. Three biological replicates were harvested, with at least 10 fruits (30 fruits at the first stage) collected from one tree per replicate. RNA-seq were performed by sequencing 250 300 bp paired-end libraries on Illumina Novaseq platforms. Sequencing were performed at the Institute of Novogene (Beijing, China). Gene expression was analyzed refer to the released 'Junzao' genome. We defined genes with reads per kilobase per million mapped reads $(\mathrm{RPKM}) \geq 1$ as being expressed.

\subsection{Salt Stress, Drought Stress, and ABA Treatment Experiments}

\subsubsection{Seedling Preparation}

Wild jujubes are genetically close to cultivated jujube and usually used as the rootstock of jujube cultivars [24]. In this study, we evaluated the expression of ZjWRKY genes of wild jujube responding to drought/salt resistance. Wild jujube seeds were collected from the State Forestry Administration Forest Tree Germplasm (Xingtai, China). Seeds were rinsed with tap water for $24 \mathrm{~h}$ and sowed in sterilized soil (peat soil: sand $=3: 1$ ). All seedlings were grown under natural light in the greenhouse of Northwest A\&F University and the temperature was $25 \pm 3^{\circ} \mathrm{C}$. Seven-week-old seedlings with similar height and biomass were selected for subsequent experiments.

\subsubsection{Drought Stress Treatment}

In first, 45 selected seedlings were transplanted from the substrates to pots. At first, the soil moisture in the pots was kept at $60 \%$ for 7 days, then stop watering and soil moisture naturally decreased. When the soil moisture reached $60 \%, 40 \%, 20 \%$, and $10 \%$, and kept for 3 days at each level, 9 seedlings were harvested, respectively. The remained 9 seedlings were re-watered. When the soil 
moisture was raised to $60 \%$ and kept for 3 days (REW60\%), the seedlings were harvested. In this experiment, the soil water content of each pot was monitored and adjusted by a weighing method [34].

\subsection{3. $\mathrm{NaCl}$ Treatment}

In total, 45 selected seedlings were transplanted from the substrates to PVC boxes containing $6 \mathrm{~L}$ $\frac{1}{2}$ Hoagland solution. Prior to $\mathrm{NaCl}$ treatment, seedlings were grown in $\frac{1}{2}$ Hoagland solution 5 days for adaptation to the hydroponic conditions. Then, the solution was replaced with $\frac{1}{2}$ Hoagland nutrient solution containing $200 \mathrm{mM} \mathrm{NaCl}$ [35]. When the treatment time reached $0,3,12,24 \mathrm{~h}, 9$ seedlings were harvested, respectively. The remaining 9 seedlings were transplanted to $\frac{1}{2}$ Hoagland nutrient solution without additional $\mathrm{NaCl}$, and harvested after $12 \mathrm{~h}$ (RS12h). Ventilation was maintained throughout hydroponic growth.

\subsubsection{ABA Treatment}

The ABA treatment was similar to the $\mathrm{NaCl}$ treatment except that $200 \mathrm{mM} \mathrm{NaCl}$ was replaced by $100 \mu \mathrm{M}$ ABA (Yuanye, Shanghai, China).

\subsection{RT-qPCR Analyses of ZjWRKY Genes in Response to Abiotic Stresses}

Gene transcription levels were quantified by RT-qPCR using TaKaRa TB Green ${ }^{\mathrm{TM}}$ Premix Ex Taq тм II (Dalian, China) on a Bio-Rad CFX Connect system (CA, USA). We made the standard curves using a 10-fold dilution series of the cDNA templates from 7-week old seedling without any treatment. The PCR amplification efficiency of each primer pair was evaluated based on the slope of a standard curve for each gene. In addition, we performed the melting curve analysis to confirm the specificity of the primer pairs. Those primers designed for tissue-expression were firstly subjected to evaluation, and we only accepted the primer sets with the amplification efficiencies ranging between $90 \%$ and $110 \%$ and melting curve showing single peak. Each $10 \mu \mathrm{L}$ reaction mixture was composed of: $5 \mu \mathrm{L}$ TB Green Premix Ex Taq II, $0.8 \mu \mathrm{L} 10 \mu \mathrm{M}$ primer set, $1 \mu \mathrm{L}$ cDNA solution, and $3.2 \mu \mathrm{L}$ ddH $_{2} \mathrm{O}$. Cycling conditions were set as $95^{\circ} \mathrm{C}$ for $30 \mathrm{~s}$ followed by 40 cycles at $95^{\circ} \mathrm{C}$ for $5 \mathrm{~s}$ and $60^{\circ} \mathrm{C}$ for $30 \mathrm{~s}$. Melting curve analysis was carried out under the following cycling conditions: $95^{\circ} \mathrm{C}$ for $5 \mathrm{~s}$ followed a gradient of $65^{\circ} \mathrm{C}$ to $95^{\circ} \mathrm{C}$ where each increase of $0.5^{\circ} \mathrm{C}$ lasted $5 \mathrm{~s}$. The expression of ZjWRKY genes was defined based on the cycle threshold $(\mathrm{Ct})$, and their relative expression levels were calculated as $2^{-\Delta \Delta \mathrm{Ct}}$ after normalization to the expression of $Z j U B Q$ as the reference gene [36]. The Venn diagram was drawn using TBtools [37].

\section{Results}

\subsection{Identification and Chromosomal Locations of ZjWRKY Family in Jujube}

In this study, a total of 61 potential ZjWRKY genes were identified through genome-wide analysis and designated according to their chromosomal location of 'Junzao' jujube genome (Table 2). All 61 predicted genes containing WRKY domains were confirmed. The length of these 61 proteins varied from 158 (ZjWRKY60) to 758 (ZjWRKY4) amino acids. The predicted MWs ranged from $18 \mathrm{kDa}$ to $82 \mathrm{kDa}$, and the pIs varied from 5.16 (ZjWRKY52) to 9.53 (ZjWRKY59). Among these $61 \mathrm{ZjWRKY}$ genes, 59 were located on the 11 jujube chromosomes, and chromosome 4 did not contain any $Z j W R K Y$ gene. $10 \mathrm{ZjWRKY}$ genes were mapped on chromosome 1, which contained the most ZjWRKY genes. Chromosomes 2 and 5 each contained only 3 ZjWRKY genes (Figure 1).

On the other hand, a total of 52 WRKY TFs were identified from the 'Dongzao' jujube genome, which distributed on 11 putative chromosomes. There was no ZjWRKY gene on chromosome 7 (Figure S1a). We found 13 members showing the same sequencing composition between the two cultivars, and 33 WRKY TFs member in 'Dongzao' have their corresponding members in 'Junzao' with high identity (>90\%). However, there were still 6 members showing low identity (53-86\%) (Table S1). 
Table 1. Identified WRKY genes in the genome of Ziziphus jujuba 'Junzao'.

\begin{tabular}{|c|c|c|c|c|c|c|c|c|}
\hline $\begin{array}{l}\text { Name } \\
\text { Symbol }\end{array}$ & Chromosome & $\begin{array}{l}\text { Peptide } \\
\text { Length }\end{array}$ & pI & MW & $\begin{array}{c}\text { CDS } \\
\text { Number }\end{array}$ & Group & $\begin{array}{c}\text { Conserved } \\
\text { Heptapeptide }\end{array}$ & $\begin{array}{l}\text { Zinc-Finger } \\
\text { Type }\end{array}$ \\
\hline ZjWRKY1 & Chr1 & 329 & 9.57 & 35 & 3 & II d & WRKYGQK & CX5CX23HXH \\
\hline ZjWRKY2 & Chr1 & 286 & 5.62 & 31 & 3 & II e & WRKYGQK & СХ5CX23HXH \\
\hline ZjWRKY3 & Chr1 & 337 & 6.37 & 37 & 3 & II c & WRKYGQK & $\mathrm{CX} 4 \mathrm{C} \times 23 \mathrm{HXH}$ \\
\hline ZjWRKY4 & Chr1 & 759 & 5.57 & 82 & 5 & I & $\begin{array}{l}\text { WRKYGQK } \\
\text { WRKYGOK }\end{array}$ & $\begin{array}{l}\mathrm{CX} 4 \mathrm{CX} 22 \mathrm{HXH} \\
\mathrm{C} 4 \mathrm{C} 23 \mathrm{HXH}\end{array}$ \\
\hline ZjWRKY5 & Chr1 & 490 & 5.70 & 52 & 3 & II e & WRKYGQK & $\mathrm{CX} 5 \mathrm{C} \times 23 \mathrm{HXH}$ \\
\hline ZjWRKY 6 & Chr1 & 634 & 6.12 & 68 & 6 & $\mathrm{II} b$ & WRKYGQK & CX5CX23HXH \\
\hline ZjWRKY7 & Chr1 & 522 & 5.12 & 57 & 2 & I & $\begin{array}{l}\text { WRKYGQK } \\
\text { WRKYGQK }\end{array}$ & $\begin{array}{l}\text { CX4CX22HXH } \\
\text { CX4CX23HXH }\end{array}$ \\
\hline ZjWRKY 8 & Chr1 & 591 & 6.12 & 65 & 7 & I & $\begin{array}{l}\text { WRKYGQK } \\
\text { WRKYGQK }\end{array}$ & $\begin{array}{l}\mathrm{CX} 4 \mathrm{CX} 22 \mathrm{HXH} \\
\mathrm{CX} 4 \mathrm{CX} 23 \mathrm{HXH}\end{array}$ \\
\hline ZjWRKY9 & Chr1 & 536 & 6.26 & 58 & 6 & II b & WRKYGQK & СХ5CX19HХН \\
\hline ZjWRKY10 & Chr1 & 317 & 8.68 & 35 & 5 & II a & WRKYGQK & CX5CX23HXH \\
\hline ZjWRKY11 & Chr2 & 648 & 6.74 & 70 & 6 & II b & WRKYGQK & CX5CX23HXH \\
\hline ZjWRKY12 & Chr2 & 200 & 9.21 & 23 & 2 & II c & WRKYGQK & CX4CX23HXH \\
\hline ZjWRKY13 & Chr2 & 648 & 6.74 & 70 & 6 & II b & WRKYGQK & CX5CX23HXH \\
\hline ZjWRKY14 & Chr3 & 346 & 6.76 & 39 & 3 & II c & WRKYGQKK & $\mathrm{CX} 4 \mathrm{CX} 23 \mathrm{HXH}$ \\
\hline ZjWRKY15 & Chr3 & 393 & 8.60 & 43 & 3 & I & $\begin{array}{l}\text { WRKYGQK } \\
\text { WRKYGQK }\end{array}$ & $\begin{array}{l}\text { CX4CX22HXН } \\
\text { CX4CX23НХН }\end{array}$ \\
\hline ZjWRKY16 & Chr3 & 619 & 6.21 & 67 & 5 & $\mathrm{II} b$ & WRKYGQ̄K & CX5CX23HXH \\
\hline ZjWRKY17 & Chr3 & 283 & 5.46 & 31 & 3 & II e & WRKYGQK & CX5CX23HXH \\
\hline ZjWRKY18 & Chr3 & 161 & 8.79 & 18 & 2 & III & WRKYGQK & $\mathrm{N}$ \\
\hline ZjWRKY19 & Chr3 & 514 & 7.63 & 56 & 4 & II b & WRKYGQK & CX5CX23HXH \\
\hline ZjWRKY20 & Chr3 & 502 & 6.52 & 55 & 4 & I & $\begin{array}{l}\text { WRKYGQK } \\
\text { WRKYGOK }\end{array}$ & CX4CX22HXH \\
\hline ZjWRKY21 & Chr5 & 398 & 7.08 & 44 & 4 & II c & WRKYGQK & CX4CX23HXH \\
\hline ZjWRKY22 & Chr5 & 560 & 5.81 & 61 & 5 & I & $\begin{array}{l}\text { WRKYGQK } \\
\text { WRKYGQK }\end{array}$ & $\begin{array}{l}\text { CX4CX22HXH } \\
\text { CX4CX23HXH }\end{array}$ \\
\hline ZjWRKY23 & Chr5 & 593 & 7.21 & 65 & 5 & I & $\begin{array}{l}\text { WRKYGQKK } \\
\text { WRKYGQK }\end{array}$ & $\begin{array}{l}\text { CX4CX22HXH } \\
\text { CX4CX23HXH }\end{array}$ \\
\hline ZjWRKY24 & Chr6 & 362 & 9.65 & 41 & 3 & II d & WRKYGQK & $\mathrm{CX} 5 \mathrm{C} \times 23 \mathrm{HXH}$ \\
\hline ZjWRKY25 & Chr6 & 336 & 6.43 & 37 & 3 & II c & WRKYGQK & $\mathrm{CX} 4 \mathrm{CX} 23 \mathrm{HXH}$ \\
\hline ZjWRKY26 & Chr6 & 558 & 7.22 & 61 & 6 & II b & WRKYGQK & CX5CX23HXH \\
\hline ZjWRKY27 & Chr6 & 290 & 5.27 & 32 & 3 & II e & WRKYGQKK & CX5CX23HXH \\
\hline ZjWRKY28 & Chr6 & 196 & 9.34 & 22 & 2 & II c & WRKYGQK & CX4CX23HXH \\
\hline ZjWRKY29 & Chr6 & 504 & 5.50 & 56 & 5 & II b & WRKYGQK & CX5CX23HXH \\
\hline ZjWRKY30 & Chr7 & 415 & 6.99 & 46 & 4 & I & $\begin{array}{l}\text { WRKYGQK } \\
\text { WRKYGQK }\end{array}$ & $\begin{array}{l}\text { CX4CX22HXH } \\
\text { CX4CX23HXH }\end{array}$ \\
\hline ZjWRKY31 & Chr7 & 311 & 5.65 & 34 & 3 & II c & WRKYGQK & CX4CX23HXH \\
\hline ZjWRKY32 & Chr7 & 636 & 6.62 & 69 & 5 & II b & WRKYGQK & CX5CX23HXН \\
\hline ZjWRKY33 & Chr7 & 632 & 6.45 & 68 & 5 & $\mathrm{II} \mathrm{b}$ & WRKYGQK & CX5CX23HXH \\
\hline ZjWRKY34 & Chr8 & 474 & 5.19 & 52 & 3 & II e & WRKYGQK & CX5CX23HXH \\
\hline ZjWRKY35 & Chr8 & 392 & 5.90 & 44 & 3 & III & WRKYGQK & CX7CX23HXC \\
\hline ZjWRKY36 & Chr8 & 345 & 5.39 & 39 & 3 & III & WRKYGQK & СХ7CХ23НХC \\
\hline ZjWRKY37 & Chr8 & 405 & 6.64 & 45 & 3 & III & WRKYGQK & CX7CX23HXC \\
\hline ZjWRKY38 & Chr9 & 393 & 4.93 & 45 & 2 & II e & WRKYGQK & CX5CX23HXH \\
\hline ZjWRKY39 & Chr9 & 543 & 7.09 & 60 & 5 & I & $\begin{array}{l}\text { WRKYGQK } \\
\text { WRKYGOK }\end{array}$ & $\begin{array}{l}\text { CX4CX22HXH } \\
\text { CX4CX23HXH }\end{array}$ \\
\hline ZjWRKY40 & Chr9 & 371 & 5.26 & 42 & 3 & III & WRKYGQ̄K & CX7CX23HXC \\
\hline ZjWRKY41 & Chr9 & 300 & 5.35 & 33 & 3 & II c & WRKYGQK & $\mathrm{CX} 4 \mathrm{CX} 23 \mathrm{HXH}$ \\
\hline ZjWRKY42 & Chr9 & 320 & 5.25 & 36 & 3 & III & WRKYGQK & СХ7CX23НХC \\
\hline ZjWRKY43 & Chr9 & 318 & 8.08 & 36 & 3 & III & WRKYGQK & CX7CX23HXC \\
\hline ZjWRKY44 & Chr9 & 308 & 5.84 & 35 & 3 & III & WRKYGQK & CX7CХ23НХC \\
\hline ZjWRKY45 & Chr9 & 304 & 5.25 & 34 & 3 & III & WRQYGQK & CX7CX23HXC \\
\hline ZjWRKY46 & Chr9 & 316 & 6.46 & 36 & 3 & III & WRKYGQK & CX7CX23HXC \\
\hline ZjWRKY47 & Chr10 & 367 & 9.64 & 39 & 3 & II d & WRKYGQK & СХ5CX23HXH \\
\hline ZjWRKY48 & Chr10 & 214 & 9.32 & 23 & 3 & II a & WRKYGQK & CX5CX23HXH \\
\hline ZjWRKY49 & Chr10 & 372 & 9.57 & 40 & 3 & II d & WRKYGQK & CX5CX23HXH \\
\hline ZjWRKY50 & Chr10 & 320 & 8.65 & 35 & 4 & II a & WRKYGQK & CX5CX23HXH \\
\hline ZjWRKY51 & Chr11 & 227 & 9.11 & 25 & 3 & II c & WRKYGQKK & $\mathrm{CX} 4 \mathrm{CX} 23 \mathrm{HXH}$ \\
\hline ZjWRKY52 & Chr11 & 374 & 5.16 & 41 & 3 & II c & WRKYGQK & CX4CX23HXH \\
\hline ZjWRKY 53 & Chr11 & 224 & 5.37 & 25 & 3 & II e & WRKYGQK & CX5CX23NXH \\
\hline ZjWRKY54 & Chr11 & 356 & 5.92 & 39 & 3 & II e & WRKYGQK & СХ5CX23HXН \\
\hline
\end{tabular}


Table 2. Identified WRKY genes in the genome of Ziziphus jujuba 'Junzao'.

\begin{tabular}{ccccccccc}
\hline $\begin{array}{c}\text { Name } \\
\text { Symbol }\end{array}$ & Chromosome & $\begin{array}{c}\text { Peptide } \\
\text { Length }\end{array}$ & pI & MW & $\begin{array}{c}\text { CDS } \\
\text { Number }\end{array}$ & Group & $\begin{array}{c}\text { Conserved } \\
\text { Heptapeptide }\end{array}$ & $\begin{array}{c}\text { Zinc-Finger } \\
\text { Type }\end{array}$ \\
\hline ZjWRKY55 & Chr11 & 478 & 9.09 & 52 & 5 & I & WRKYGQK & CX4CX22HXH \\
ZjWRKY56 & Chr12 & 243 & 8.66 & 28 & 3 & II c & WRKYGQK & CX4CX23HXH \\
ZjWRKY57 & Chr12 & 196 & 6.73 & 22 & 3 & II c & WRKYGKK & CX4CX23HXH \\
ZjWRKY58 & Chr12 & 355 & 5.23 & 41 & 3 & III & WRKYGQK & CX7CX23HXH \\
ZjWRKY59 & Chr12 & 329 & 9.53 & 36 & 3 & II d & WRKYGQK & CX5CX23HXH \\
ZjWRKY60 & Conting12.1 & 159 & 5.46 & 18 & 3 & II c & WRKYGKK & CX4CX23HXH \\
ZjWRKY61 & Conting111 & 237 & 7.65 & 26 & 4 & II c & WRKYGQK & CX4CX23HXH \\
\hline
\end{tabular}

CDS number: the number of coding sequences.

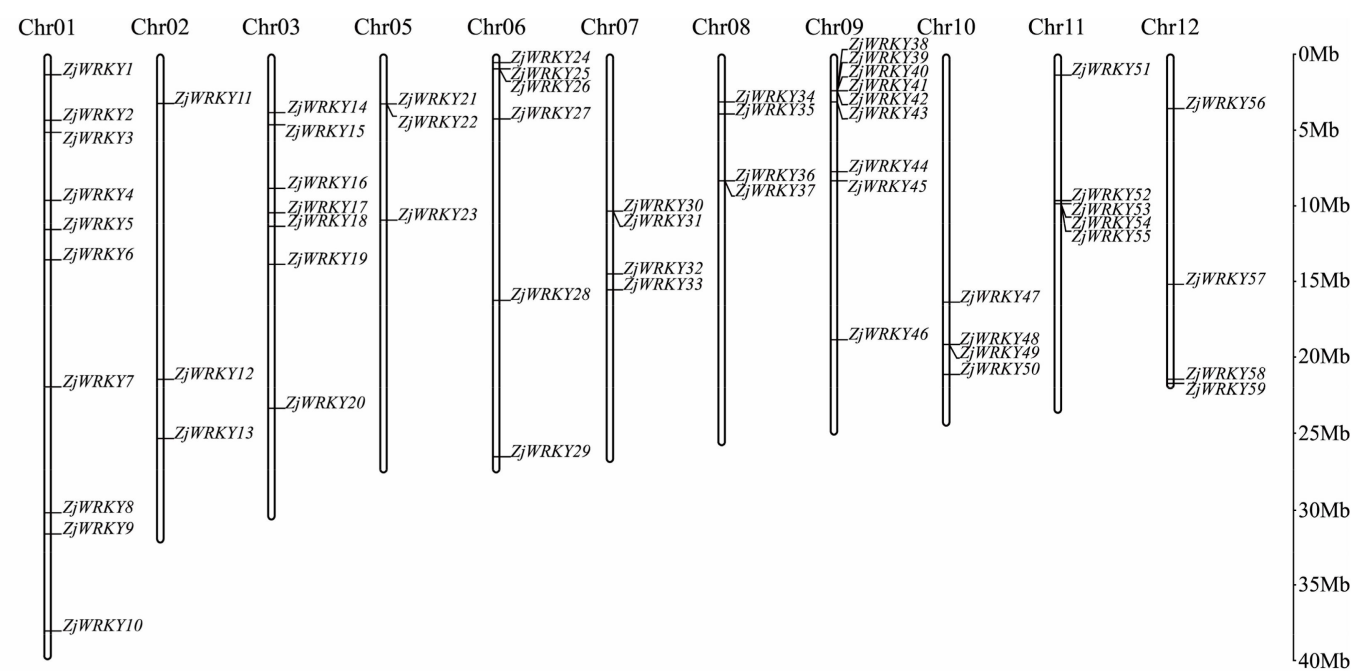

Conting12.1Conting111

Figure 1. Distribution of $Z j W R K Y$ genes on pseudo chromosomes of 'Junzao'. The scale on the right is in million bases $(\mathrm{Mb})$.

\subsection{Phylogenetic Classification of ZjWRKY Genes}

ZjWRKY TFs of 'Junzao' jujube were classified into three groups based on the number of WRKY domains and the type of zinc finger motif. Group I contained two WRKY motifs (one in the N-terminal region of the sequence, the other in the C-terminal region) and two $\mathrm{C}_{2} \mathrm{H}_{2}$ zinc finger motifs; 10 ZjWRKYs were assigned to this group. Group II contained a WRKY motif and a $\mathrm{C}_{2} \mathrm{H}_{2}$ zinc finger motif, except for ZjWRKY53. This group contained 40 members. Group III included 11 members, and each of them contained a WRKY motif followed with a $\mathrm{C}_{2} \mathrm{HC}$ zinc finger motif, except ZjWRKY18 (Figure 2 and Table 2). On the other hand, 52 WRKY TFs of 'Dongzao' jujube were also divided into 3 groups which were group I with 10 members, group II with 30 members, group III with 12 members. In contrast to 'Junzao', WRKY TFs in group II of 'Dongzao' was obviously reduced (Table S2 and Figure S1b).

For further classification, WRKY proteins from three different plant species, including 71 from A. thaliana, 77 from S. lycopersicum, and 61 from Z. jujuba 'Junzao', were subjected to phylogenetic analysis. Group II was divided into five subgroups (IIa to IIe) according to the zinc finger structure (Figure 2 and Figure S2). Subgroups IIa, IIb, IIc, IId, and IIe contained 3, 10, 14, 5, and 8 members, respectively. In 'Dongzao' jujube, subgroups IIa, Ilb, IIc, IId, and IIe contained 2, 8, 12, 3, and 5 members, respectively (Table S2). The zinc finger structure from the group IN was C- $\mathrm{X}_{4}-\mathrm{C}-\mathrm{X}_{22}-\mathrm{H}-\mathrm{X}_{1}-\mathrm{H}$, and IC was $\mathrm{C}-\mathrm{X}_{4}-\mathrm{C}-\mathrm{X}_{23}-\mathrm{H}-\mathrm{X}_{1}-\mathrm{H}$. The members of subgroups IIa, IIb, IId, and IIe had C- $\mathrm{X}_{5}-\mathrm{C}-\mathrm{X}_{23}-\mathrm{H}-\mathrm{X}_{1}-\mathrm{H}$ zinc-finger motifs, except for the ZjWRKY53. Group IIc had C- $\mathrm{X}_{4}-\mathrm{C}-\mathrm{X}_{23}-\mathrm{H}-\mathrm{X}_{1}-\mathrm{H}$ zinc finger structure. Group III 
had a $\mathrm{C}-\mathrm{X}_{7}-\mathrm{C}-\mathrm{X}_{23}-\mathrm{H}-\mathrm{X}_{1}-\mathrm{C}$ zinc finger motif, except for ZjWRKY18, which had no complete zinc-finger structure (Figure S3). We found 4 members (XP_015874714.1, XP_024928884.1, XP_024933427.1, and XP_015902857.1) in group III did not have a complete zinc finger structure in 'Dongzao' jujube (Figure S1g).

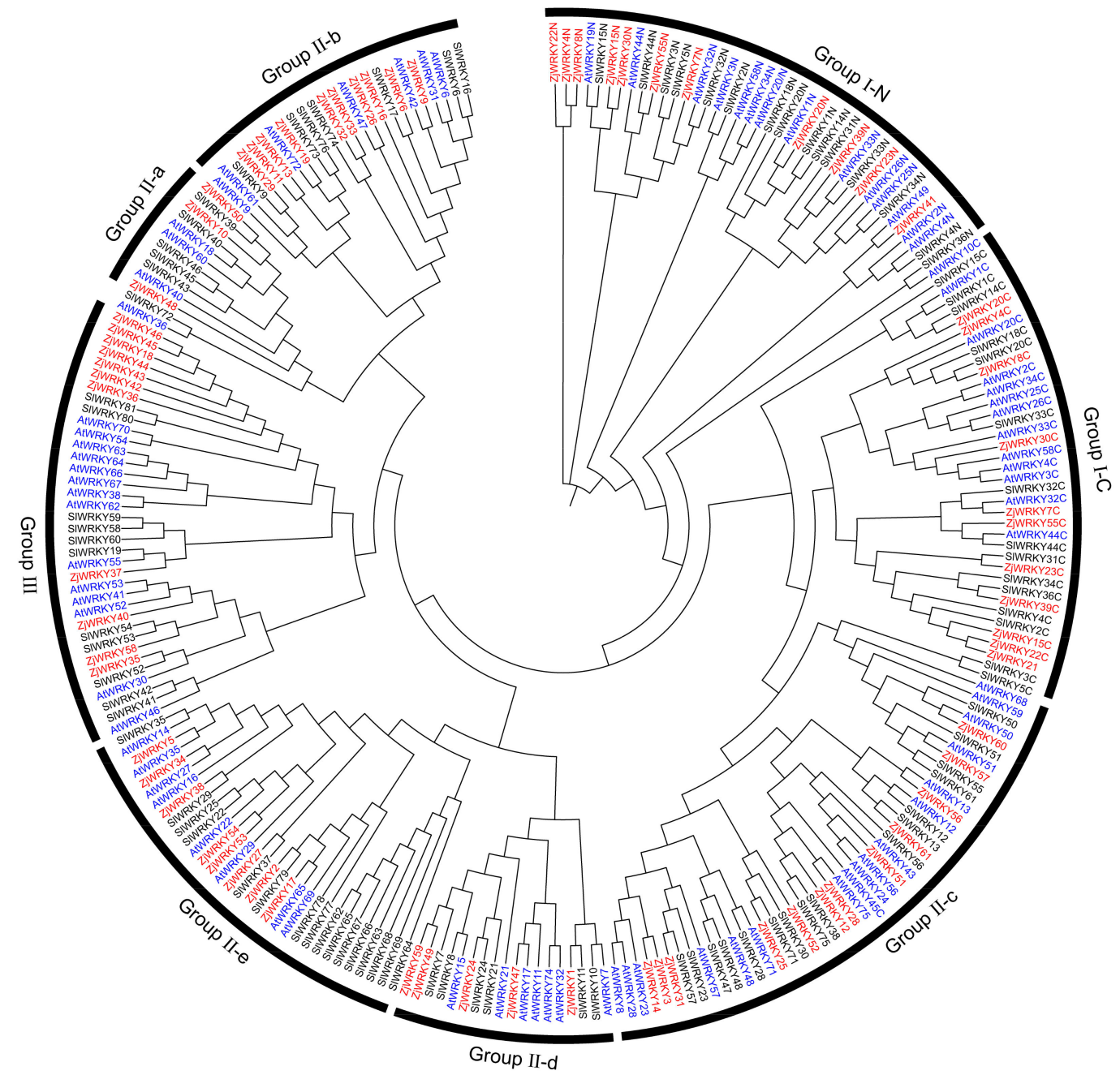

Figure 2. Phylogenetic analysis of the WRKY proteins from Ziziphus jujuba 'Junzao' jujube, Arabidopsis thaliana and Solanum lycopersicum. Multiple sequence alignments of WRKY amino acid sequences were performed using ClustalW. The phylogenetic tree was constructed using MEGA7.0 with the neighbor-joining method and 1000 bootstrap replicates (substitution model: Jones-Taylor-Thornton model). Group (I, II, III) and subgroup (IIa III) names were marked outside the circle.

Although the WRKY domain was highly conserved, there were some mutations in Chinese jujube. The WRKY motifs (WRKYGQK) had one amino acid modification (WRKYGKK) in ZjWRKY57 and ZjWRKY60 that belonging to group IIc, whereas in ZjWRKY45 (group III), the WRKY domain was replaced by WRQY (Figure S3). In ‘Dongzao' jujube, group IIc (XP_015884439.1 and XP_015870123.1) also contained the aa sequence WRKYGKK. The mutations in the conserved domain of these WRKY genes have been confirmed by sanger sequencing in 'Junzao' jujube. In addition, we identified the orthologous genes (AtWRKY51 and AtWRKY50) of A. thaliana regarding to ZjWRKY57 and ZjWRKY60 by phylogenetic analyses and also found the mutation (WRKYGKK) in these proteins. However, we did not find an orthologous gene of ZjWRKY45 in A. thaliana and S. lycopersicum. 


\subsection{Conserved Motifs of the ZjWRKY and Structure of Their Genes}

MEME motif analysis identified 10 motifs in all WRKY members of two Chinese jujube cultivars. Each ZjWRKY had different motif combinations. Motif 1 was annotated as WRKY DNA-binding motif, which is the fundamental characteristic of WRKY TFs (Figure S4) and presented in all ZjWRKY at least one time (Figure 3 and Figure S1c). Motif 2 was zinc finger motif and almost presented in all ZjWRKYs except two members (ZjWRKY18 and XP 015902857.1) belonging to group III. Group I proteins had two WRKY domains, each consisting of the conserved aa sequence WRKYGQK (motif 1) and a zinc finger (motif 2), except XP 015892560.1 of 'Dongzao'. Motif 5 was unique to group Ilb. Motif 6 only presented in group IIb of 'Dongzao' jujube while it presented in both group IIb and group IIc (ZjWRKY61) of 'Junzao' jujube.

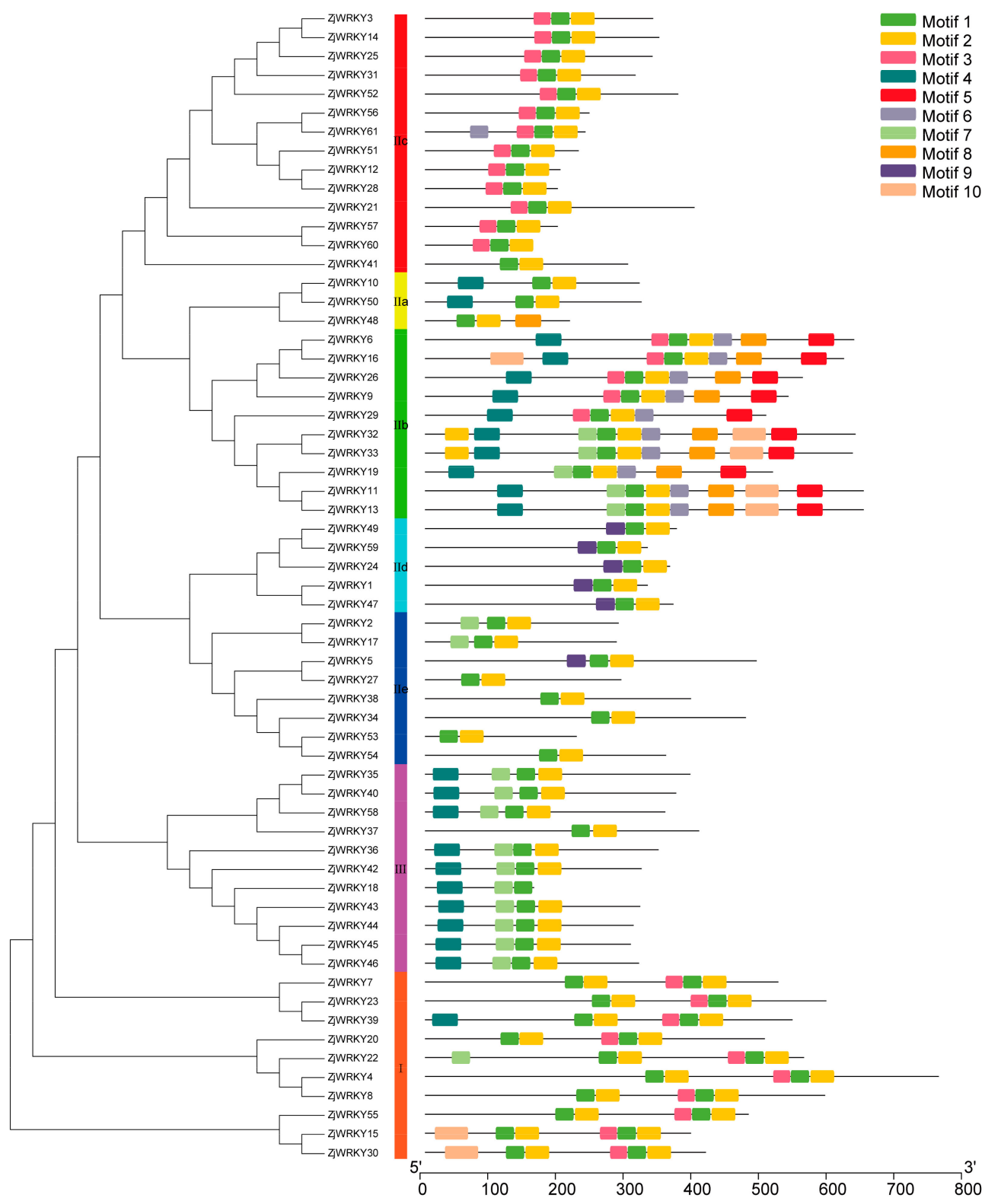

Figure 3. Phylogenetic tree and motif composition of 'Junzao' WRKY proteins. The phylogenetic tree was constructed using MEGA7.0 with the neighbor-joining method and 1000 bootstrap replicates (substitution model: Jones-Taylor-Thornton model). Multiple Em for Motif Elicitation (MEME) was used to predict motifs. Ten motifs were identified and were represented by different colors. The position of the motif on the chromosome was labeled. 
Gene structure predictions revealed that all ZjWRKY genes contained CDS and introns. The number of CDS varied from 2 to 7. ZjWRKY genes containing 3 CDS were the most frequent type and accounted for $55.74 \%$ (Figure 4). In similar, ZjWRKY containing 3 CDS was the most dominant type in 'Dongzao' jujube (Figure S1d).
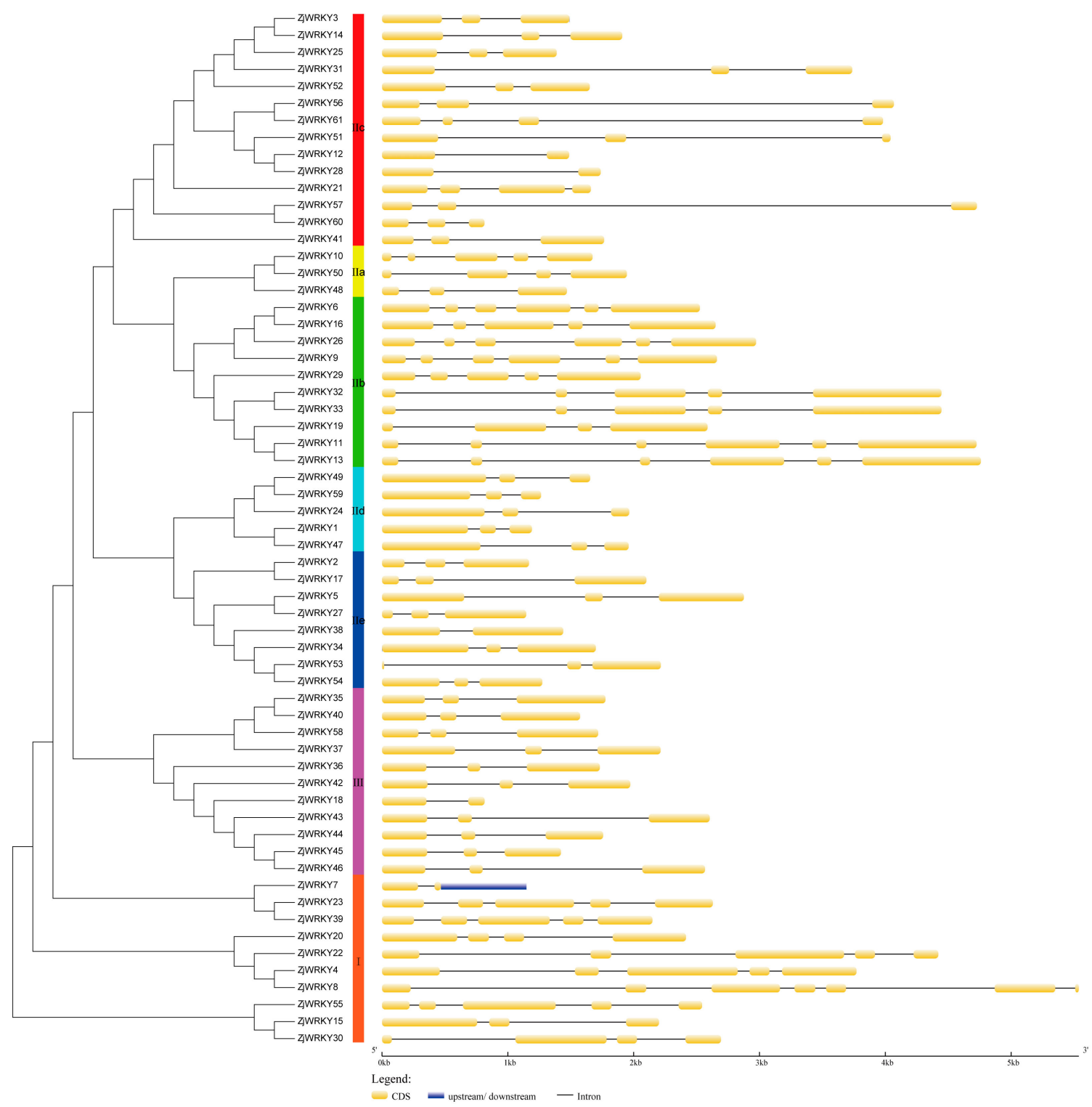

Figure 4. Intron-exon structure of ZjWRKY genes of Ziziphus jujuba 'Junzao'. The phylogenetic tree was constructed using MEGA7.0 with the neighbor-joining method and 1000 bootstrap replicates (substitution model: Jones-Taylor-Thornton model). Analysis of ZjWRKY genes structure using gene structure display server (GSDS). Yellow blocks indicate the coding sequences (CDS), blue blocks indicate upstream or downstream sequences, and black lines indicate introns.

\subsection{Cis-Acting Regulatory Elements of the ZjWRKY Promoters}

These cis-acting regulatory elements in the 'Junzao' ZjWRKY genes promoter regions were mainly classified into four categories: light-responsive elements, hormone-responsive elements, stress-responsive elements, and growth and metabolic-responsive elements (Figure 5a,b). The light-responsive elements were present in the promoter regions of all $\mathrm{ZjWRKY}$, and included Box 4, G-box, and GT1-motif. Hormone-responsive elements included response elements to ABA (ABRE), auxin (AuxRR-core, and TGA-box), gibberellin (GARE-motif, P-box, and TATC-box), methyl 
jasmonate (CGTCA-motif, and TGACG-motif), and salicylic acid (TCA-element), among which the methyl jasmonate (MeJA)-responsive elements were the most dominant, accounting for $37.36 \%$ of the hormone-responsive elements. The stress-responsive elements included response elements to anaerobic induction (ARE), defense and stress (TC-rich repeats), drought (MBS), low temperature (LTR) and wound (WUN-motif). Among these, response elements to anaerobic induction were the most abundant, accounting for $55.00 \%$ of stress-responsive elements. We found hormone-responsive and stress-responsive elements present in the promoter region of some $Z j W R K Y$ genes in each of the three groups. Growth and metabolic-responsive elements mainly include: elements associated with Zein-metabolism regulation $\left(\mathrm{O}_{2}\right.$-site), meristem expression (CAT-box), and endosperm expression (GCN4_motif). In addition, we found the W-box element in the promoter regions of the $43 \mathrm{ZjWRKY}$ genes (Table S3). Similar to the 'Junzao' jujube, the light-responsive elements were observed in the promoter region of all 'Dongzao' jujube WRKY genes. Unlike the 'Junzao' jujube, the ABA-responsive elements were the most dominant among the hormone-responsive elements, and cis-acting regulatory element involved in zein metabolism regulation was the most dominant among the growth and metabolic-responsive elements (Figure S1e,f).

\subsection{Tissue-Specific Expression Profile of ZjWRKY Genes}

To investigate whether the predicted 'Junzao' WRKY genes were actually transcribed, we examined their transcription levels in different tissues using RT-PCR. We designed primer sets for each candidate ZjWRKY genes (Table S4). RT-PCR analyses showed that 41 genes were transcribed in roots, stems, flowers, leaves, young fruit and ripe fruit, but their expression levels varied among these tissues (Figure 6). As shown in Figure 6, seven genes (ZjWRKY19, 20, 23, 24, 39, 47, and 52) highly expressed in all six tissues. Further analysis of the promoter regions of these 7 genes revealed that ABA-responsive element was the dominant type followed by anaerobic induction and SA-responsive element (Table S3). No bands were detected in all tissues for two genes (ZjWRKY18, and 46). ZjWRKY46 was activated by the following drought, $\mathrm{NaCl}$ and $\mathrm{ABA}$ treatments while $\mathrm{ZjWRKY18}$ was still not detected under such treatments (Figure S5). We identified 1 ABA-responsive element, 1 SA-responsive element and 1 defense and stress-responsive element in the promoter region of ZjWRKY46 and 2 MeJA-responsive elements, 1 ABA-responsive element and 1 defense and stress-responsive element in the promoter region of ZjWRKY18 (Table S3). ZjWRKY32, and 33 in group IIe were specifically expressed in roots. There were 4 MeJA-responsive elements, 4 anaerobic induction-responsive elements and 2 low temperature-responsive elements in the promoter regions of these 2 genes. $Z j W R K Y 41$, in group IIc, was specifically expressed in young fruit (15 DAF), and ZjWRKY43, and 45, in group III, were only detected in flowers. We found 1 GA-responsive element was common in the promoter regions of ZjWRKY41, 43 and 45 (Table S3).

We further examined the expression patterns of ZjWRKY 43 and 45 in floral organs, including sepals, disks, pistils, and stamen, by RT-PCR. ZjWRKY43 was expressed in pistils and disks, and ZjWRKY45 was expressed in pistils, sepals and disks. Neither were expressed in the stamen (Figure S6). In addition, the transcription levels of $15 \mathrm{ZjWRKY}$ genes (ZjWRKY9, 12, 14, 25, 28, 29, 34, 38, 41, 44, 51, 55, 57, 60, and 61 ) changed between young and ripe fruits (Table S5). 
A
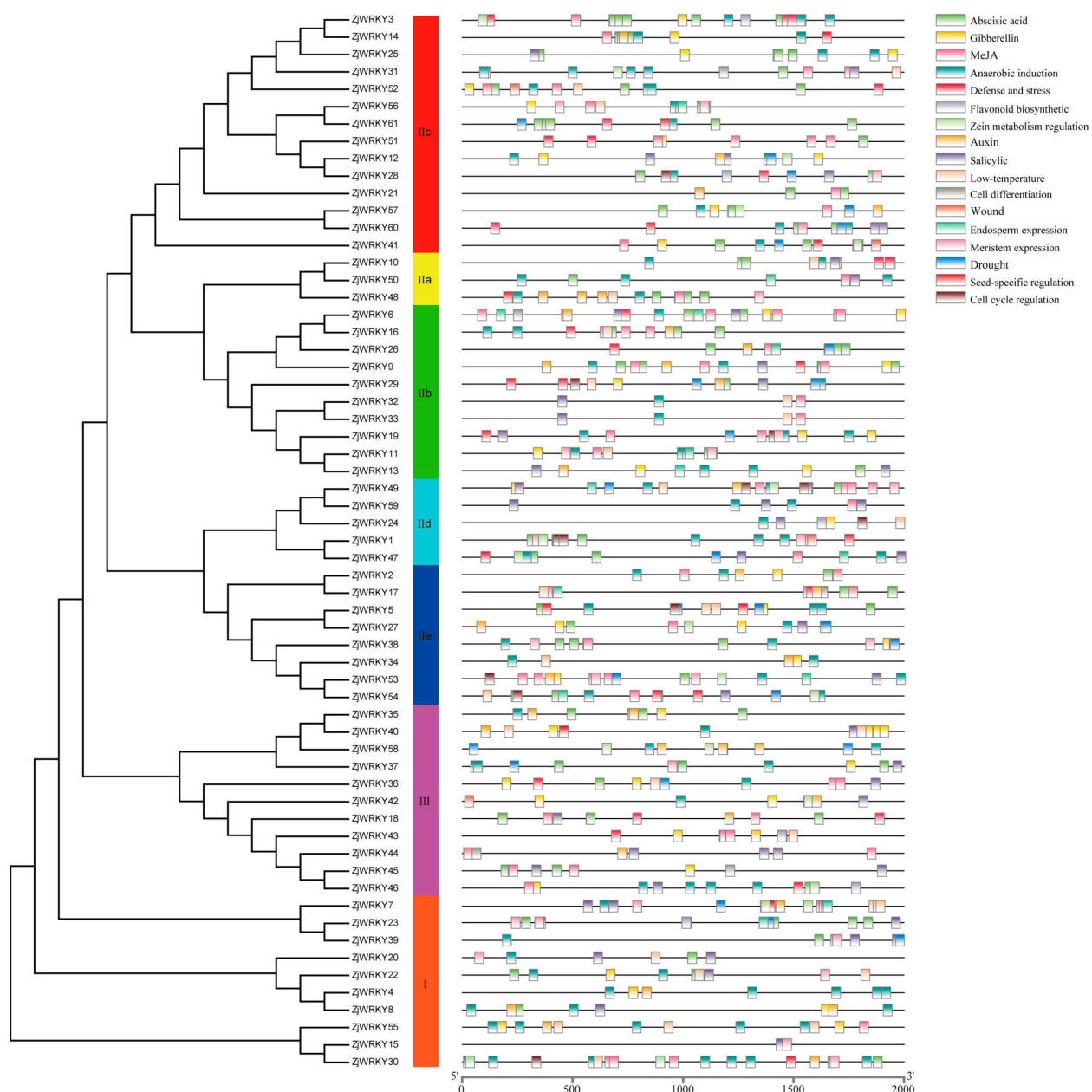

B

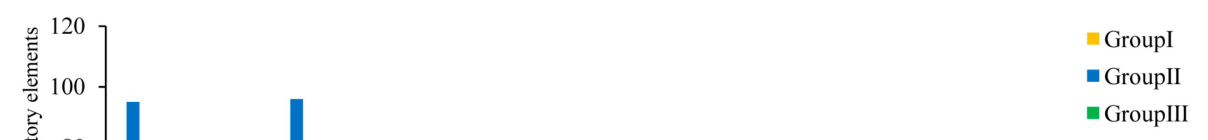

Hormone

Stress

Growth and metabolism

Figure 5. Type and number of cis-acting regulatory elements in the promoter region of WRKY genes of Ziziphus jujuba 'Junzao'. (A) Position of the cis-acting elements in the ZjWRKY genes promoter region. (B) Number of cis-acting elements. 


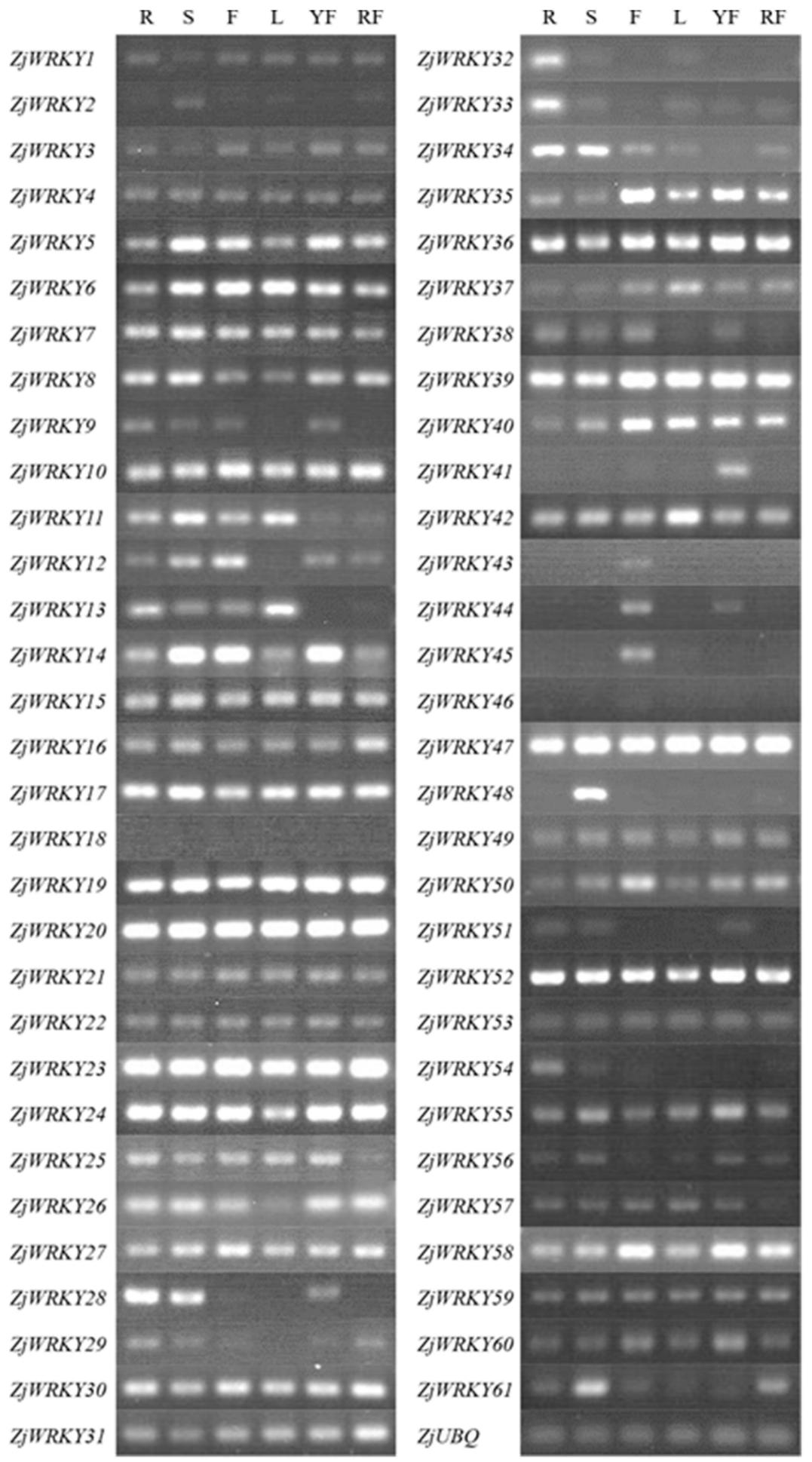

Figure 6. RT-PCR analysis of ZjWRKY genes in six types of tissues/organs of 'Junzao' jujube. R: root; S: stem; F: flower; L: leaf; YF: young fruit; and RF: ripe fruit.

\subsection{Transcriptional Dynamics of ZjWRKY Genes During Fruit Development and Ripening}

Wild jujube is the wild type of jujube and genetically close to jujube, so their WRKY TFs might be similar with high possibility. On the other hand, wild jujube fruit is smaller and more acidic than jujube [24]. In order to identify ZjWRKY genes that might involve in fruit enlargement and sugar accumulation, we detected the expression level of $Z j W R K Y$ genes during the fruits development of wild and cultivated jujube. Based on the transcriptomic analysis, in total, there were 39 and 44 
ZjWRKY genes with RPKM value $\geq 1$ during fruit development and ripening of 'Junzao' jujube and 'Qingjiansuanzao' wild jujube, respectively. These 39 genes expressed in 'Junzao' fruit were divided into 5 clusters based on their expression level (Figure 7). In cluster 1 ( 9 genes), the expression level increased gradually. In cluster 2 (7 genes), the expression level decreased gradually. In cluster 3 (5 genes), the expression level increased first and then decreased, whereas in cluster 4 (8 genes), the expression level decreased first and then increased. In cluster 5 (10 genes), expression level was variable.

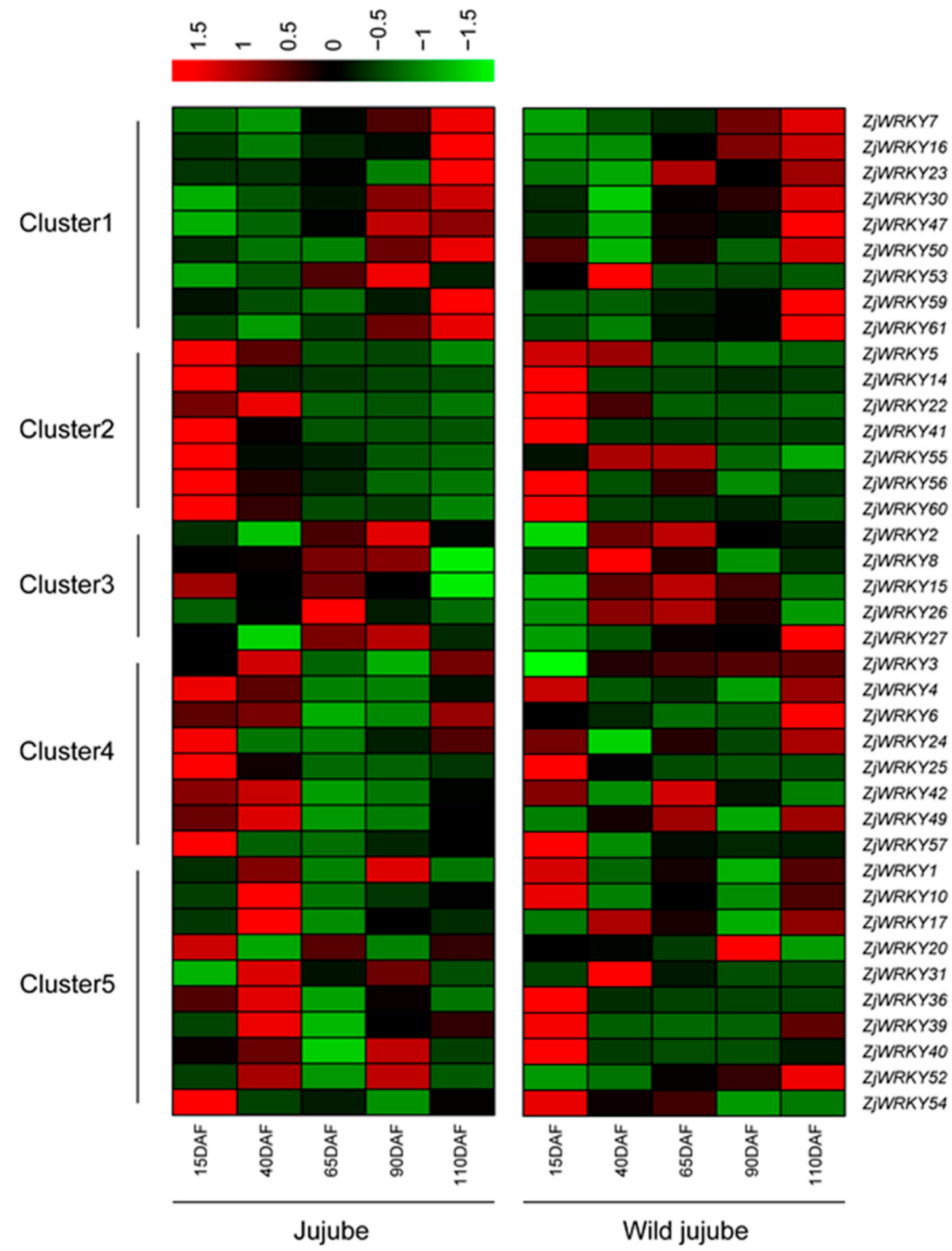

Figure 7. Transcriptional abundance of ZjWRKY genes during fruit ripening of cultivated jujube ('Junzao') and wild jujube ('Qingjiansuanzao'). RPKM was used to measure the expression levels of the ZjWRKY genes. Red denotes high expression levels, and green denotes low expression levels. Clustering was done according to the expression level of ZjWRKY genes in jujube fruit.

We further compared the transcript abundance of ZjWRKY genes between 'Junzao' and 'Qingjiansuanzao' during fruit ripening. We found that 20 of these 39 ZjWRKY genes showed similar 
expression patterns between the two accessions during the progress of fruit ripening. Additionally, the transcript abundance of 7 genes (ZjWRKY5, 24, 25, 30,41, 57, and 60) in 'Junzao' fruit was always higher than that in 'Qingjiansuanzao' fruit, whereas the transcript abundance of 12 genes $(Z j W R K Y 1,2$, $3,6,14,15,16,26,49,52,54$, and 55) in 'Junzao' fruit was always lower than those of 'Qingjiansuanzao'. In addition, we found that ZjWRKY genes belonging to group I and group IId had a higher transcript abundance $(R P K M \geq 10)$ than those genes in the other groups.

\subsection{Transcriptional Responses of ZjWRKY Genes to Salt, and Drought Stresses and ABA Treatment}

To accurately evaluate the relative expression level of ZjWRKY genes by qRT-PCR analyses, we first established a standard curve for each gene to confirm the amplification efficiency of the primer sets. Among the 61 ZjWRKY genes, the transcription levels of 6 genes $(Z j W R K Y 9,18,22,43,45$, and 46) were too low in all organs/tissues to establish standard curves.

Under drought stress condition (10\% water content), 21 ZjWRKY genes in the wild jujube seedlings were upregulated by over 2-fold, and two of them (ZjWRKY37, and 48) increased >10-fold (Figure 8a). In particular, ZjWRKY37 (group III) exhibited the highest expression level, with $>30$-fold upregulation. Analysis of cis-acting elements displayed that there were 2 ABA-responsive elements and 1 SA-responsive element in the promoter region of ZjWRKY37 and there were 5 ABA-responsive elements and 2 MeJA-responsive elements in the promoter region of ZjWRKY48. In contrast, the expression levels of 9 ZjWRKY genes exhibited >2-fold decreases in these seedlings (Figure 8a).

Under salt stress condition ( $200 \mathrm{mM} \mathrm{NaCl}$ for $24 \mathrm{~h}$ ), expression levels of $8 \mathrm{ZjWRKY}$ genes showed $>2$-fold increases in the wild jujube seedlings. Among these genes, ZjWRKY27 exhibited the highest expression level, with >5-fold increases. There were 3 MeJA-responsive elements, 1 SA-responsive element and 1 drought-responsive element in the promoter region of ZjWRKY27. On the other hand, 5 ZjWRKY genes exhibited >2-fold downregulation in these seedlings (Figure 8b).

For seedlings treated with $100 \mu \mathrm{M}$ ABA for $24 \mathrm{~h}$, the expression of $10 \mathrm{ZjWRKY}$ genes were $>2$-fold upregulated and ZjWRKY23 was mostly upregulated (group I), with >18-fold increases in the seedlings. In the ZjWRKY23 promoter region, we identified 3 ABA-responsive elements, 1 SA-responsive element and 1 drought-responsive element. On the other hand, $8 \mathrm{ZjWRKY}$ genes were downregulated by $>2$-fold in these seedlings (Figure 8c).

We selected some genes which were regulated by $>2$-fold at transcript level after drought, salt and ABA treatment for further analysis. Under drought stress, as shown in Figure S7a, the maximum value of expression of different ZjWRKY genes appeared in different degrees of drought stress (Figure S8). It is worth noting that the expression levels of 5 genes $(Z j W R K Y 27,28,30,37,48$, and 56) were negatively correlated with soil water content, that is, the expression level of these genes increased when the soil moisture reduced, but the expression level fell after rehydration (Figure 9a). 


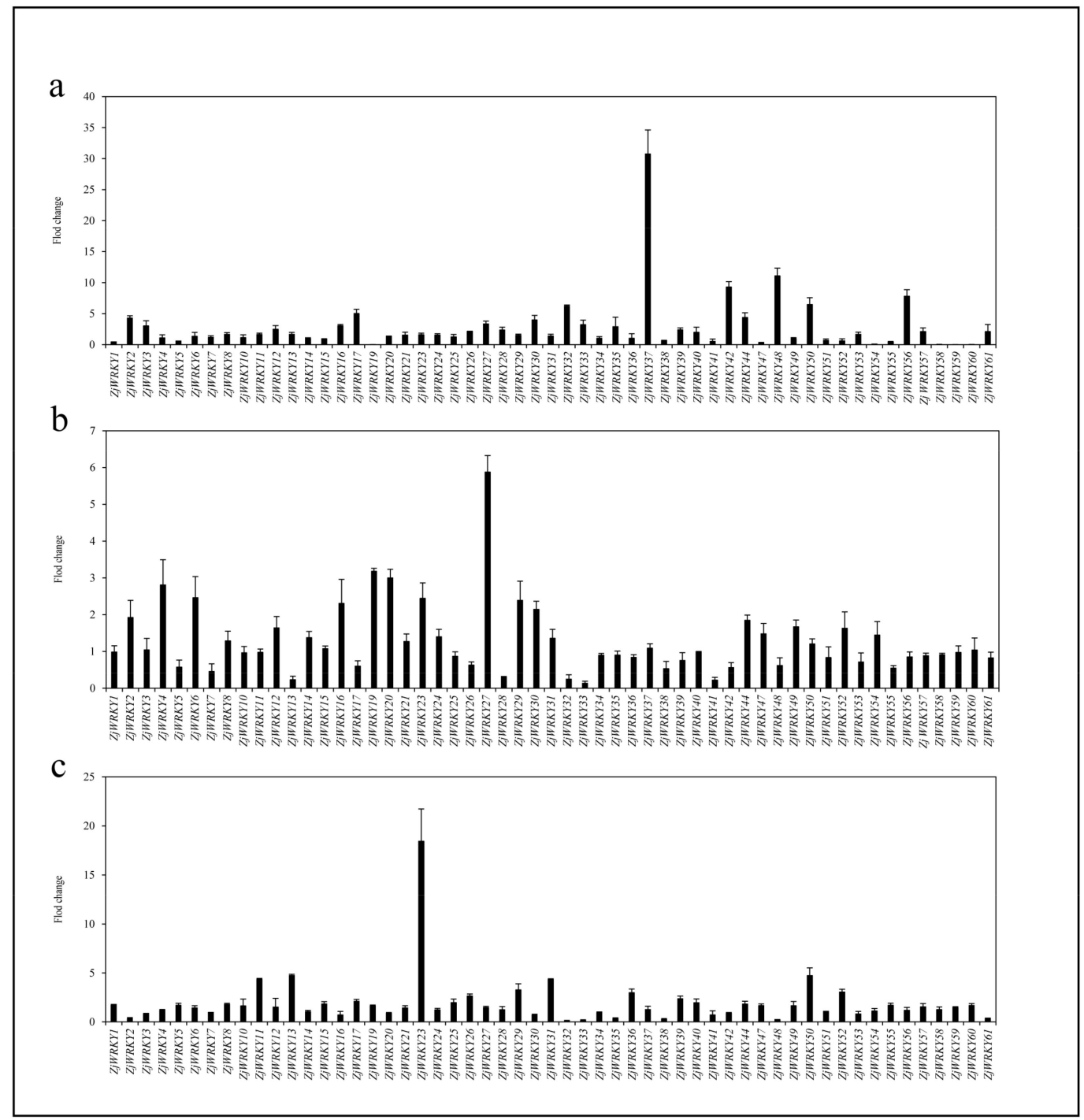

Figure 8. ZjWRKY genes expression in wild jujube seedlings under three different treatments. (a) Expression level of ZjWRKY genes under drought stress; (b) Expression level of ZjWRKY genes under $\mathrm{NaCl}$ stress; (c) Expression level of ZjWRKY genes under ABA treatment. The histograms represent the fold changes of expression level compared to the reference. The error bars represent standard deviation. Z $j W R K Y$ genes expression level were determined by real-time quantitative polymerase chain reaction (RT-qPCR) using $Z j U B Q$ as a positive reference.

Among the selected 14 ZjWRKY genes (Figure S7b), there were 2, 1, 0, and 8 genes reaching their highest transcript level when treated with $\mathrm{NaCl}$ for $0,3,12$, and $24 \mathrm{~h}$, respectively. The transcription level of ZjWRKY27 increased with the time of $\mathrm{NaCl}$ treatment and fell back after removal of $\mathrm{NaCl}$. This pattern was similar with that under drought treatment (Figure 9b). ZjWRKY4, and 29 firstly decreased to their lowest level after 1 to $3 \mathrm{~h} \mathrm{NaCl}$, and then increased with the time of $\mathrm{NaCl}$ treatment, and fell back after removal of $\mathrm{NaCl}$.

Under ABA treatment, the expression level of ZjWRKY23 was positively correlated with the duration of ABA treatment. There were 6 ZjWRKY genes (ZjWRKY17, 26, 29, 36, 39, and 52) that showed similar trends, except their transcription levels decreased during the first $3 \mathrm{~h}$ of ABA treatment. Interestingly, $\mathrm{ZjWRKY} 29$ showed a similar trend under $\mathrm{NaCl}$ and $\mathrm{ABA}$ treatments (Figure 9c). 
a

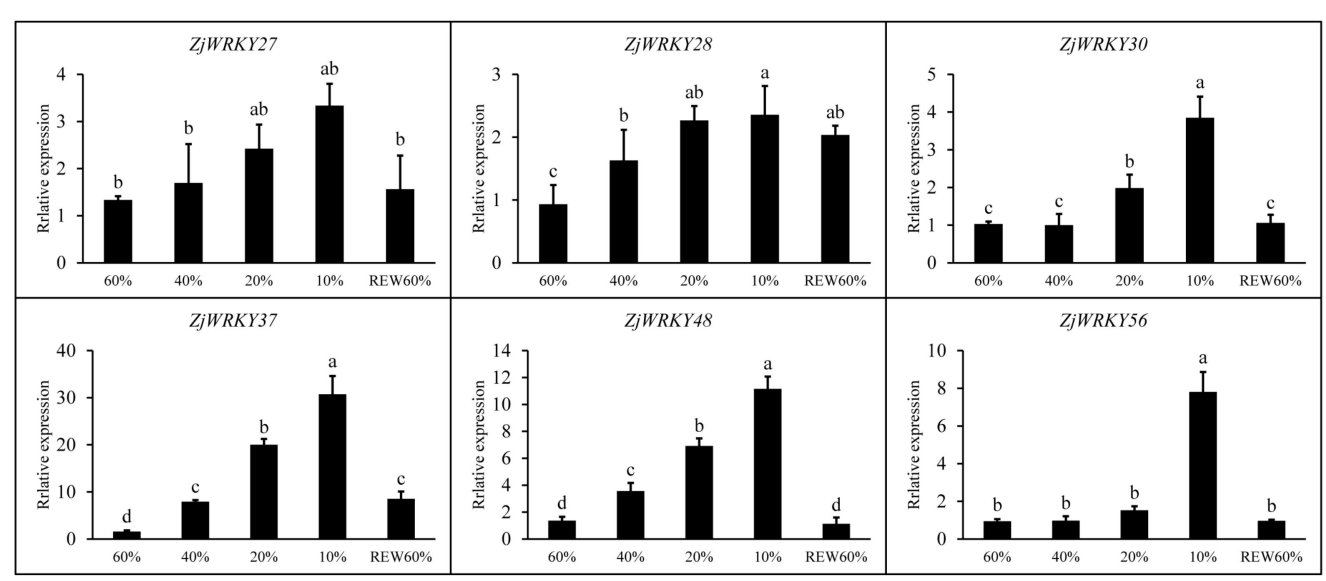

$\mathrm{b}$

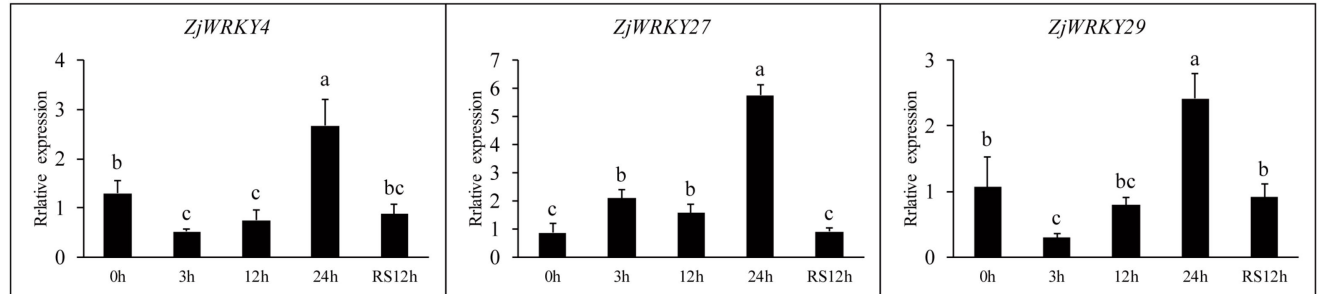

C

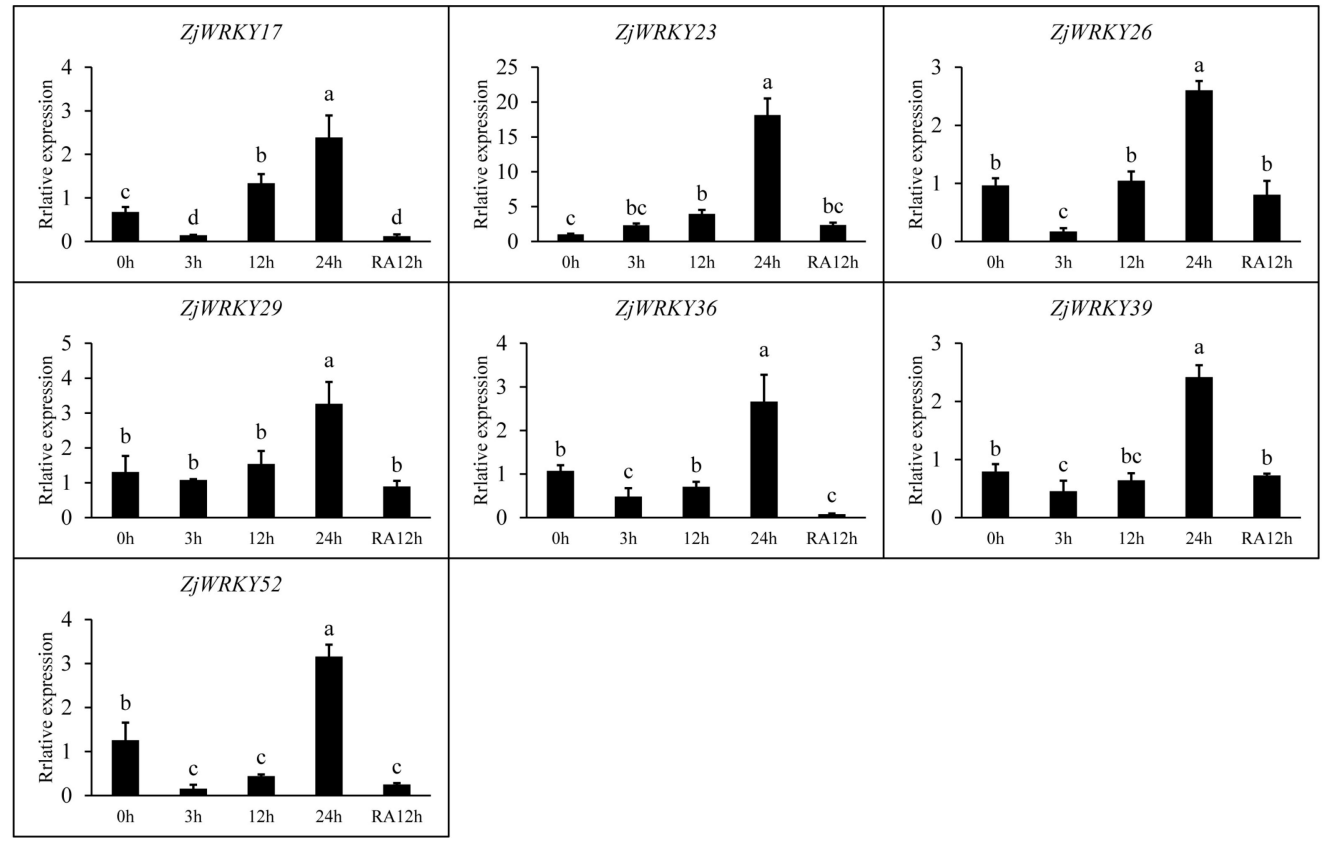

Figure 9. Expression profile of the $Z j W R K Y$ genes under three treatments. (a) Expression level of 6 ZjWRKY genes in soil moisture content $60 \%, 40 \%, 20 \%, 10 \%$, and $60 \%$ after re-watering (REW60\%); (b) Expression level of $3 \mathrm{ZjWRKY}$ genes under $0 \mathrm{~h}, 3 \mathrm{~h}, 12 \mathrm{~h}$, and $24 \mathrm{~h} \mathrm{NaCl}$ treatment, and $12 \mathrm{~h}$ removal of $\mathrm{NaCl}$ (RS 12 h); (c) Expression level of $7 \mathrm{ZjWRKY}$ genes after $0 \mathrm{~h}, 3 \mathrm{~h}, 12 \mathrm{~h}$, and $24 \mathrm{~h}$ of ABA treatment, and $12 \mathrm{~h}$ after removal of ABA (RA $12 \mathrm{~h}$ ). The histograms represent the relative expression level of $Z j W R K Y$ genes compared to the reference. Error bars represent standard deviations. The data were calculated using the method of $2^{-\Delta \Delta C t}$. LSD detection was used to label different letters when there was a significant difference in expression $(p<0.05 ; n=3)$.

Taken together, we constructed a Venn diagram of ZjWRKY genes involved in fruit development and response to drought, $\mathrm{NaCl}$ and ABA treatment. As showed in Figure 10, there were $39 \mathrm{ZjWRKY}$ 
genes expressed during 'Junzao' jujube fruit development, and 30, 12, 18 ZjWRKY genes responded to drought, $\mathrm{NaCl}$, and ABA treatments, respectively. Some ZjWRKY genes were involved in several processes. For example, $29 \mathrm{ZjWRKY}$ genes expressed during the fruit development were also responded to abiotic stress. Among which, expression levels of ZjWRKY32 and 33 in wild jujube seedlings changed when exposed to drought, $\mathrm{NaCl}$ and $\mathrm{ABA}$ treatments (Table S6). However, no genes were expressed in all events.

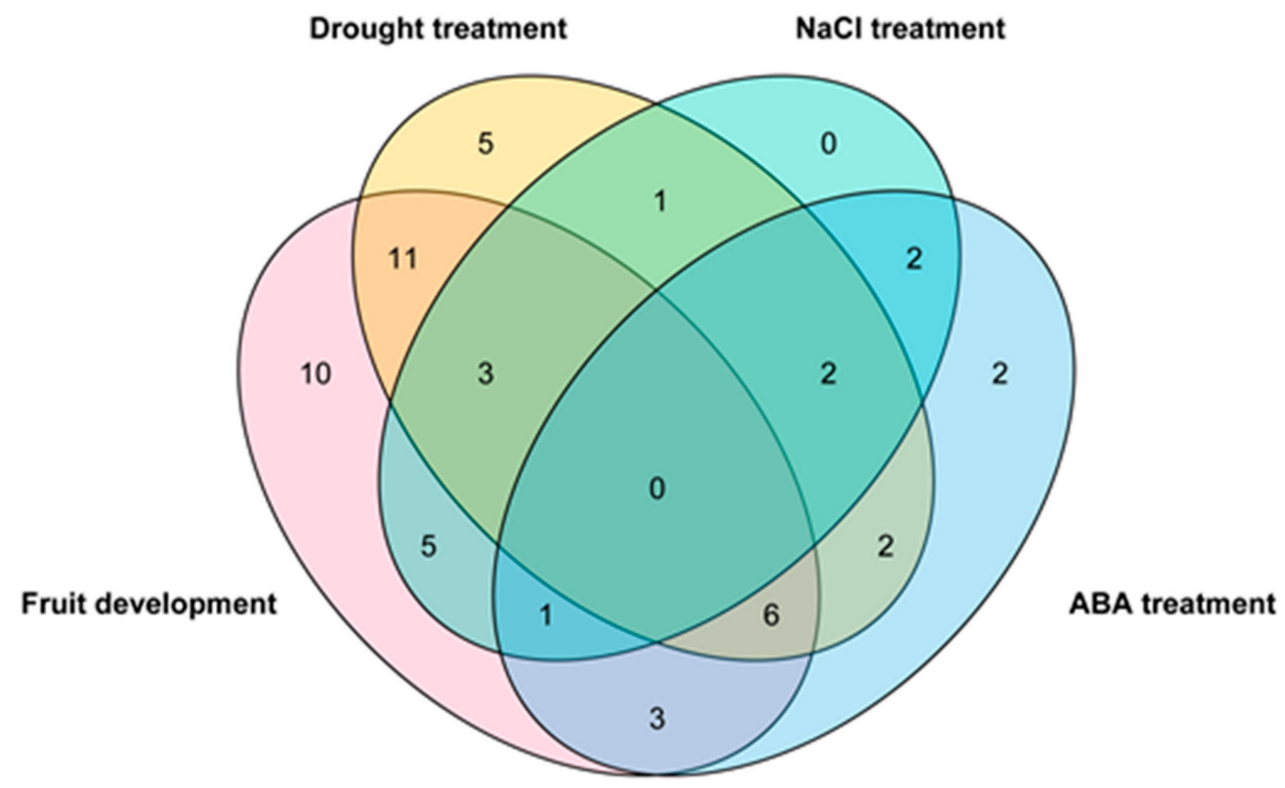

Figure 10. The Venn diagram of ZjWRKY genes related to fruit development, drought, $\mathrm{NaCl}$, and ABA treatment.

\section{Discussion}

\subsection{Identification of the WRKY Family Members in Chinese Jujube}

Accompanying the increasing number of plant genomes which were resolved, WRKY gene family, an important transcription factors family, has been identified in more and more plant species. In this study, we identified a total of 61 and 52 WRKY TFs from the 'Junzao' and 'Dongzao' jujube genome, respectively. Although most WRKY genes from 'Dongzao' have their corresponding members in 'Junzao' genome, there were some members that did not share between two accessions (Table S1). This difference also indicated more alleles existed among different cultivars. Of course, the difference in the number of WRKY TFs between 'Junzao' and 'Dongzao' might be also attributed to the difference in cultivar or sequencing strategy.

In contrast, there were 127 WRKY TFs in M. domestica belonging to Rosaceae that is neighbor with Rhamnaceae, about twice as large as in Z. jujuba [14]. This difference was contributed by the recent whole genome duplication event in the apple genome while no such event occurred in jujube genome [24].

\subsection{ZjWRKY Expression Profile in Different Tissues}

We analyzed the expression pattern of the ZjWRKY genes of 'Junzao' jujube in six different tissues. The results demonstrated variation in the expression patterns of ZjWRKY genes. In total, 7 genes were highly expressed in all jujube tissues. Highly expressed WRKY genes usually play important roles in plant development [23]. Therefore, we concluded that these 7 highly expressed ZjWRKY genes might be important regulatory factors in jujube development, although further studies are required to verify the function of these genes. In contrast, 2 ZjWRKY genes were expressed at low levels in all jujube tissues and 6 ZjWRKY genes were specifically expressed in only one tissue. Some low expression genes were 
induced by the specific environment. For example, ZjWRKY46 was not detected in normal growing plants, but it was detected in seedlings treated with drought, $\mathrm{NaCl}$, and ABA. However, ZjWRKY18 cannot be detected in all samples. It might be activated by other conditions. Genes expressed at high levels in special organs usually play key roles in plant development [38]. In A. thaliana, AtWRKY34 and $A t W R K Y 2$ have higher expression levels in pollen and pollen tubes compared to other tissues. They were required for the development of male gametophytes [19]. In jujube, ZjWRKY32, and 33 were specifically expressed in roots. Their homologous gene, AtWRKY72, has a higher expression level in the lateral root cap and epidermis compared to other tissues [39]. AtWRKY72 contributes to basal immunity in A. thaliana [40]. ZjWRKY43 and ZjWRKY45 were expressed only in flowers, ZjWRKY48 was specifically expressed in stems, and ZjWRKY41 was specifically expressed in young fruit. They might have specific functions in those corresponding tissues.

\subsection{ZjWRKY Genes Associated with Jujube Fruit Development and Ripening}

Fruit development and ripening is a complex process that includes cell division and expansion, the accumulation of sugar, fruit coloration, and other physiological processes. Studies have shown that a variety of TFs were involved in fruit development and ripening [41,42]. In pepper, CaWRKY1 expression was strongly up-regulated in red fruit and may play an important role in fruit maturation [43]. In banana, the protein MaHIS1 and MaWRKY1 could interact with and regulate physiological processes like fruit ripening and stress responses [44]. In this study, we found $39 \mathrm{ZjWRKY}$ genes expressed during jujube fruit development and ripening. The genes of groups I and IId had higher expression levels than those of other groups, which indicated ZjWRKY genes belonging to group I and IId play more important roles than other groups in jujube fruit development. Similar results as described above were also observed during the development of pepper fruit [23]. In this study, expression levels of $7 \mathrm{ZjWRKY}$ genes were gradually decreased during the process of fruit ripening. We found the cis-acting regulatory element that responds to gibberellin signal from the promoter region of these genes. Moreover, the gibberellin content gradually decreased during the ripening of the 'Dongzao' jujube fruit [45]. Therefore, we speculate that these genes may be related to gibberellin.

Considering the huge change in fruit size and fruit taste between jujube and wild jujube, WRKY TFs might play a role in the difference of fruit ripening. By comparing ZjWRKY member expression patterns between jujube and wild jujube, we found that 7 ZjWRKY genes display higher transcript levels in jujube fruit than in wild jujube fruit while $12 \mathrm{ZjWRKY}$ genes showed higher transcript levels in wild jujube fruit. Therefore, these ZjWRKY might control genes involved in fruit enlargement and quality development.

\subsection{ZjWRKY Genes Involved in Response to Abiotic Stresses}

WRKY TFs are widely involved in the regulation of plant responses to stress. In A. thaliana, M. domestica and Sesamum indicum, 18, 34, and 26 WRKY genes were associated with salt, waterlogging and drought stresses, respectively $[14,34,46]$. In this study, we found that 30, 14, and $18 \mathrm{ZjWRKY}$ genes responded to drought stress, salt stress and ABA treatment, respectively. WRKY TFs could regulate plant responses to abiotic stress through ABA-dependent pathways. In A. thaliana, AtWRKY46 modulates the development of $A$. thaliana lateral roots in osmotic/salt stress conditions via regulation of ABA signaling [47]. In this study, ZjWRKY29 had a similar expression pattern with salt and ABA treatment, so this gene may respond to salt stress through ABA-dependent pathway. In addition, we found that ZjWRKY27 had similar expression patterns under drought stress and salt stress (Figure 9a,b), indicates that it can function in jujube under different stresses. Previous studies have demonstrated that single transcription factor may function in several seemingly disparate signaling pathways. For example, AtWRKY33 reacted to both heat treatment and salt treatment [48].

There were 5 genes $(Z j W R K Y 27,28,30,37,48$, and 56) responding positively to drought stress. Among these 5 genes, ZjWRKY48 is the orthology gene of AtWRKY40. In A. thaliana, ABA induction of AtWRKY18 and AtWRKY40 leads to increase in WRKY18 and WRKY40 proteins that 
form heterocomplexes through physical interactions. WRKY18/WRKY40 heterocomplex could activate AtWRKY60 expression. AtWRKY60 positively regulate plant responses to ABA [49]. Thus, some of them may play an important role in jujube response to drought stress.

Upon ABA treatment, the expression level of ZjWRKY23 increased with ABA treatment. AtWRKY33 is an orthologs of ZjWRKY23, which could upregulated in A. thaliana by ABA treatment [50]. Overexpression of $A t W R K Y 33$ can increase $\mathrm{NaCl}$ tolerance in $A$. thaliana [51]. In addition, the expression level of $Z j W R K Y 27$ increased with the duration of $\mathrm{NaCl}$ treatment. How those ZjWRKY genes regulating jujube responding to ABA needs be further elucidated by gene functional analyses.

In addition, according to the distribution pattern of cis-regulation elements in the promoter regions of ZjWRKY TFs, we found the distribution of cis-regulatory elements in the promotor regions of different ZjWRKY TFs was unnecessarily related with the gene expression pattern. Thus, their potential roles in gene regulation need to be validated in future research.

\section{Conclusions}

In this study, we identified and compared 61 and 52 ZjWRKY TFs from two famous jujube cultivars, i.e., 'Junzao' and 'Dongzao', respectively. We characterized their distribution on chromosomes, conserved motifs, gene structure, cis-acting regulatory elements in promoter regions, and evolutionary relationships. This difference of 'Junzao' and 'Dongzao' ZjWRKY TFs might be attributed to the difference in sequencing strategies or genetic background of two cultivars. Furthermore, we found ZjWRKY genes of 'Junzao' showed different expression pattern in the different tissues. Among the 61 genes, 7 ZjWRKY genes highly expressed in various tissues. Transcriptome analysis showed that 39 ZjWRKY genes expressed during 'Junzao' fruit ripening, among which 19 ZjWRKY genes were differentially expressed between 'Junzao' and 'Qingjiansuanzao', indicating their possible roles regulating sugar accumulation and acid metabolism. On the other hand, expression levels of 30, 14 , and $18 \mathrm{ZjWRKY}$ genes changed when wild jujube seedlings were exposed to drought, $\mathrm{NaCl}$ and ABA treatments, respectively. Among these genes, 5 and 1 ZjWRKY genes were strongly correlated with the degree of drought and salt stress, respectively. Taken together, our study revealed the functional diversity of WRKY TFs and provided candidate WRKY genes for future breeding of drought/salt-tolerance jujube trees.

Supplementary Materials: The following are available online at http://www.mdpi.com/2073-4425/10/5/360/s1. Figure S1: The WRKY genes identification, evolutionary relationships, motif characteristics, genes structure, and cis-acting element types and numbers of WRKY TFs from 'Dongzao' jujube. Figure S2: Phylogenetic analysis of the WRKY proteins from Chinese jujube, Arabidopsis thaliana and Solanum lycopersicum. Multiple sequence alignments of WRKY amino acid sequences were performed using ClustalW. The phylogenetic tree was constructed using MEGA7.0 with the Maximum Likelihood method. Figure S3: Multiple sequence alignment of 'Junzao' WRKY conserved domains. Dashed lines indicate the gap. The overall height of each stack on the logo illustrates the sequence conservation at each position. Each residue letter height symbolizes the relative frequency of the corresponding residue. Figure S4: Detailed information of the 'Junzao' and 'Dongzao' motif obtained by Multiple Em for Motif Elicitation (MEME). Figure S5: Expression levels of ZjWRKY18, and 46 after drought, $\mathrm{NaCl}, \mathrm{ABA}$ treatments. Figure S6: Expression level of ZjWRKY43, and 45 in sepals, disks, pistils, and stamen. Figure S7: Expression profile of the ZjWRKY genes under three treatments. a: Expression level of $30 \mathrm{ZjWRKY}$ genes in soil moisture content $60 \%, 40 \%, 20 \%, 10 \%$, and $60 \%$ after re-watering (REW60\%); b: Expression level of 14 ZjWRKY genes under $0 \mathrm{~h}, 3 \mathrm{~h}, 12 \mathrm{~h}$, and $24 \mathrm{~h} \mathrm{NaCl}$ treatment, and $12 \mathrm{~h}$ removal of $\mathrm{NaCl}$ (RS $12 \mathrm{~h}$ ); c: Expression level of $18 \mathrm{ZjWRKY}$ genes after $0 \mathrm{~h}, 3 \mathrm{~h}, 12 \mathrm{~h}$, and $24 \mathrm{~h}$ of ABA treatment, and $12 \mathrm{~h}$ after removal of ABA (RA $12 \mathrm{~h}$ ). The histograms represent the relative expression level of $Z j W R K Y$ genes compared to the reference. Error bars represent standard deviations. The data were calculated using the method of $2^{-\Delta \Delta C t}$. LSD detection was used to label different letters when there was a significant difference in expression $(p<0.05 ; \mathrm{n}=3)$. Figure S8: Growth status of wild jujube seedlings under drought stress. Table S1: Comparison of ZjWRKY protein sequences of 'Dongzao' and 'Junzao' using BLAST-2.3.31+. Table S2: Identified WRKY genes in the genome of Ziziphus jujuba 'Dongzao'. Table S3: Identified cis-acting regulatory elements in the putative promoter regions of 'Junzao' WRKY genes. Table S4: Designed primer sets used in this study. Table S5: Relative expression levels of the ZjWRKY genes in six tissues/organs of 'Junzao' jujube using Image J. Table S6: ZjWRKY genes related to fruit development, drought, $\mathrm{NaCl}$ and $\mathrm{ABA}$ treatment.

Author Contributions: Conceptualization, J.H., Y.W., and C.W.; methodology, X.C., and R.C.; software, X.C.; validation, R.C., Y.W. and C.W.; formal analysis, X.C., R.C. and J.H.; investigation, J.H.; resources, J.H., C.W.; 
data curation, J.H.; writing-original draft preparation, X.C., R.C.; writing-review and editing, C.W., J.H., Y.W.; visualization, X.C.; supervision, J.H.; project administration, H.J.; funding acquisition, C.W., J.H. and Y.W.

Funding: This work was supported by the Key Project of Science and Technology of Xinjiang Production and Construction Corps (Grant No.2017DB006-1), National Natural Science Foundation of China (Grant No.31751005, 31870584), and Cyrus Tang Foundation to Dr. Jian Huang.

Acknowledgments: We are grateful to Xingang Li and Anzhi Wei from College of Forestry, Northwest A\&F University for providing the facility of laboratory and greenhouse.

Conflicts of Interest: The authors declare no conflict of interest.

\section{References}

1. Liu, M.J.; Wang, J.R.; Liu, P.; Zhao, J.; Zhao, Z.H.; Dai, L.; Li, X.S.; Liu, Z.G. Historical achievements and frontier advances in the production and research of Chinese jujube (Ziziphus jujuba) in China. Acta Hortic. Sin. 2015, 42, 1683-1698. [CrossRef]

2. Song, S.; Zhou, H.; Sheng, S.; Cao, M.; Li, Y.; Pang, X. Genome-wide organization and expression profiling of the SBP-box gene family in Chinese jujube (Ziziphus jujuba Mill.). Int. J. Mol. Sci. 2017, 18, 1734. [CrossRef] [PubMed]

3. Zhang, J. China Forestry Statistical Yearbook; China Forestry Publishing House: Beijing, China, 2014; p. 474.

4. Zhang, P.; Shi, Y.J.; Song, F.H.; Zhuo, R.T.; Wu, Z.B. Investigation on variation and correlation of the main nutrition quality traits of Ziziphus jujuba cv. Huizao from south of Xinjiang. J. Fruit Sci. 2011, 28, 77-81.

5. Liu, M.J.; Zhao, J.; Cai, Q.L.; Liu, G.C.; Wang, J.R.; Zhao, Z.H.; Liu, P.; Dai, L.; Yan, G.; Wang, W.J.; et al. The complex jujube genome provides insights into fruit tree biology. Nat. Commun. 2014, 5, 5315. [CrossRef] [PubMed]

6. Golldack, D.; Luking, I.; Yang, O. Plant tolerance to drought and salinity: Stress regulating transcription factors and their functional significance in the cellular transcriptional network. Plant. Cell Rep. 2011, 30, 1383-1391. [CrossRef]

7. Zhu, J.K. Abiotic stress signaling and responses in plants. Cell 2016, 167, 313-324. [CrossRef]

8. Zhang, C.; Wang, D.; Yang, C.; Kong, N.; Shi, Z.; Zhao, P.; Nan, Y.; Nie, T.; Wang, R.; Ma, H.; et al. Genome-wide identification of the potato WRKY transcription factor family. PLoS ONE 2017, 12, e0181573. [CrossRef]

9. Silva Monteiro de Almeida, D.; Oliveira Jordao do Amaral, D.; Del-Bem, L.E.; Bronze Dos Santos, E.; Santana Silva, R.J.; Peres Gramacho, K.; Vincentz, M.; Micheli, F. Genome-wide identification and characterization of cacao WRKY transcription factors and analysis of their expression in response to witches' broom disease. PLoS ONE 2017, 12, e0187346. [CrossRef]

10. Wang, L.; Zhu, W.; Fang, L.; Sun, X.; Su, L.; Liang, Z.; Wang, N.; Londo, J.P.; Li, S.; Xin, H. Genome-wide identification of WRKY family genes and their response to cold stress in Vitis vinifera. BMC Plant Biol. 2014, 14, 103. [CrossRef]

11. Chu, X.; Wang, C.; Chen, X.; Lu, W.; Li, H.; Wang, X.; Hao, L.; Guo, X. The cotton WRKY gene GhWRKY41 positively regulates salt and drought stress tolerance in transgenic Nicotiana benthamiana. PLoS ONE 2015, 10, e0157026. [CrossRef]

12. Duan, G.F.; Li, L.J.; Liu, Q.L. A WRKY transcription factor from Malus domestica negatively regulates dehydration stress in transgenic Arabidopsis. Acta Physiol. Plant 2014, 36, 541-548. [CrossRef]

13. Zhang, Y.; Yu, H.; Yang, X.; Li, Q.; Ling, J.; Wang, H.; Gu, X.; Huang, S.; Jiang, W. CsWRKY46, a WRKY transcription factor from cucumber, confers cold resistance in transgenic-plant by regulating a set of cold-stress responsive genes in an ABA-dependent manner. Plant Physiol. Biochem. 2016, 108, 478-487. [CrossRef]

14. Meng, D.; Li, Y.; Bai, Y.; Li, M.; Cheng, L. Genome-wide identification and characterization of WRKY transcriptional factor family in apple and analysis of their responses to waterlogging and drought stress. Plant Physiol. Biochem. 2016, 103, 71-83. [CrossRef]

15. Vives-Peris, V.; Marmaneu, D.; Gómez-Cadenas, A.; Perez-Clemente, R.M. Characterization of Citrus WRKY transcription factors and their responses to phytohormones and abiotic stresses. Biol. Plantarum. 2017, 62, 1-12. [CrossRef] 
16. Dai, W.; Wang, M.; Gong, X.; Liu, J.H. The transcription factor FcWRKY40 of Fortunella crassifolia functions positively in salt tolerance through modulation of ion homeostasis and proline biosynthesis by directly regulating SOS2 and P5CS1 homologs. New Phytol. 2018, 219, 972-989. [CrossRef]

17. Zhu, D.; Che, Y.; Xiao, P.; Hou, L.; Guo, Y.; Liu, X. Functional analysis of a grape WRKY30 gene in drought resistance. Plant Cell Tiss. Org. 2018, 132, 449-459. [CrossRef]

18. Miao, Y.; Laun, T.; Zimmermann, P.; Zentgraf, U. Targets of the WRKY53 transcription factor and its role during leaf senescence in Arabidopsis. Plant Mol. Biol. 2004, 55, 853-867. [CrossRef]

19. Guan, Y.; Meng, X.; Khanna, R.; LaMontagne, E.; Liu, Y.; Zhang, S. Phosphorylation of a WRKY transcription factor by MAPKs is required for pollen development and function in Arabidopsis. PLoS Genet. 2014, 10, e1004384. [CrossRef]

20. Johnson, C.S.; Kolevski, B.; Smyth, D.R. TRANSPARENT TESTA GLABRA2, a trichome and seed coat development gene of Arabidopsis, encodes a WRKY transcription factor. Plant Cell 2002, 14, 1359-1375. [CrossRef]

21. Suttipanta, N.; Pattanaik, S.; Kulshrestha, M.; Patra, B.; Singh, S.K.; Yuan, L. The transcription factor CrWRKY1 positively regulates the terpenoid indole alkaloid biosynthesis in Catharanthus roseus. Plant Physiol. 2011, 157, 2081-2093. [CrossRef]

22. Goel, R.; Pandey, A.; Trivedi, P.K.; Asif, M.H. Genome-wide analysis of the Musa WRKY gene family: Evolution and differential expression during development and stress. Front. Plant Sci. 2016, 7, 299. [CrossRef]

23. Yuan, C.; Ahammed, G.J.; Yu, J.; Yao, Z.; Ruan, M.; Ye, Q.; Li, Z.; Wang, R.; Feng, K.; Zhou, G.J.S.R. Putative $W R K Y$ s associated with regulation of fruit ripening revealed by detailed expression analysis of the WRKY gene family in pepper. Sci. Rep. 2016, 6, 39000.

24. Huang, J.; Zhang, C.; Zhao, X.; Fei, Z.; Wan, K.; Zhang, Z.; Pang, X.; Yin, X.; Bai, Y.; Sun, X. The jujube genome provides insights into genome evolution and the domestication of sweetness/acidity taste in fruit trees. PLoS Genet. 2016, 12, e1006433. [CrossRef]

25. Finn, R.D.; Coggill, P.; Eberhardt, R.Y.; Eddy, S.R.; Mistry, J.; Mitchell, A.L.; Potter, S.C.; Punta, M.; Qureshi, M.; Sangrador Vegas, A.; et al. The Pfam protein families database: Towards a more sustainable future. Nucleic Acids Res. 2016, 44, D279-D285. [CrossRef]

26. Johnson, L.S.; Eddy, S.R.; Portugaly, E. Hidden Markov model speed heuristic and iterative HMM search procedure. BMC Bioinform. 2010, 11, 431. [CrossRef]

27. Larkin, M.A.; Blackshields, G.; Brown, N.P.; Chenna, R.; McGettigan, P.A.; McWilliam, H.; Valentin, F.; Wallace, I.M.; Wilm, A.; Lopez, R.; et al. Clustal W and Clustal X version 2.0. Bioinformatics 2007, 23, 2947-2948. [CrossRef]

28. Kumar, S.; Stecher, G.; Tamura, K. MEGA7: Molecular evolutionary genetics analysis version 7.0 for bigger datasets. Mol. Biol. Evol. 2016, 33, 1870. [CrossRef]

29. Bailey, T.L.; Boden, M.; Buske, F.A.; Frith, M.; Grant, C.E.; Clementi, L.; Ren, J.; Li, W.W.; Noble, W.S. MEME Suite: Tools for motif discovery and searching. Nucleic Acids Res. 2009, 37, W202-W208. [CrossRef]

30. Hu, B.; Jin, J.; Guo, A.Y.; Zhang, H.; Luo, J.; Gao, G. GSDS 2.0: An upgraded gene feature visualization server. Bioinformatics 2015, 31, 1296-1297. [CrossRef]

31. Postel, D.; Vanlemmens, P.; Gode, P.; Ronco, G.; Villa, P. PlantCARE, a database of plant cis-acting regulatory elements and a portal to tools for in silico analysis of promoter sequences. Nucleic Acids Res 2002, 31, 325-327.

32. Zhang, C.; Huang, J.; Li, X. Identification of appropriate reference genes for RT-qPCR analysis in Ziziphus jujuba Mill. Sci. Hortic. Amst. 2015, 197, 166-169. [CrossRef]

33. Abràmoff, M.D.; Magalhães, P.J.; Ram, S.J. Image processing with ImageJ. Biophotonics Int. 2004, 11, $36-42$.

34. Li, D.; Liu, P.; Yu, J.; Wang, L.; Dossa, K.; Zhang, Y.; Zhou, R.; Wei, X.; Zhang, X. Genome-wide analysis of WRKY gene family in the sesame genome and identification of the WRKY genes involved in responses to abiotic stresses. BMC Plant Biol. 2017, 17, 152. [CrossRef]

35. Matsuura, H.; Ishibashi, Y.; Shinmyo, A.; Kanaya, S.; Kato, K.J.P. Genome-wide analyses of early translational responses to elevated temperature and high salinity in Arabidopsis thaliana. Plant Cell Physiol. 2010, 51, 448-462. [CrossRef]

36. Livak, K.J.; Schmittgen, T.D. Analysis of relative gene expression data using real-time quantitative PCR and the 2- $\Delta \Delta$ CT method. Methods 2001, 25, 402-408. [CrossRef] 
37. Chen, C.J.; Xia, R.; Chen, H.; He, Y.H. TBtools, a Toolkit for Biologists integrating various biological data handling tools with a user-friendly interface. BioRxiv 2018, 289660.

38. Ramamoorthy, R.; Jiang, S.Y.; Kumar, N.; Venkatesh, P.N.; Ramachandran, S. A comprehensive transcriptional profiling of the WRKY gene family in rice under various abiotic and phytohormone treatments. Plant Cell Physiol. 2008, 49, 865-879. [CrossRef]

39. Birnbaum, K.; Shasha, D.E.; Wang, J.Y.; Jung, J.W.; Lambert, G.M.; Galbraith, D.W.; Benfey, P.N.J.S. A gene expression map of the Arabidopsis root. Science 2003, 302, 1956-1960. [CrossRef]

40. Bhattarai, K.K.; Atamian, H.S.; Kaloshian, I.; Eulgem, T. WRKY72-type transcription factors contribute to basal immunity in tomato and Arabidopsis as well as gene-for-gene resistance mediated by the tomato $R$ gene Mi-1. Plant J. 2010, 63, 229-240. [CrossRef]

41. Fujisawa, M.; Nakano, T.; Shima, Y.; Ito, Y. A large-scale identification of direct targets of the tomato MADS box transcription factor RIPENING INHIBITOR reveals the regulation of fruit ripening. Plant Cell 2013, 25, 371-386. [CrossRef]

42. Lee, S.; Chung, E.J.; Joung, Y.H.; Choi, D. Non-climacteric fruit ripening in pepper: Increased transcription of EIL-like genes normally regulated by ethylene. Funct. Integr. Genomic 2010, 10, 135-146. [CrossRef]

43. Marchive, C.; Mzid, R.; Deluc, L.; Barrieu, F.; Pirrello, J.; Gauthier, A.; Corio-Costet, M.-F.; Regad, F.; Cailleteau, B.; Hamdi, S.J.J.o.E.B. Isolation and characterization of a Vitis vinifera transcription factor, VvWRKY1, and its effect on responses to fungal pathogens in transgenic tobacco plants. J. Exp. Bot. 2007, 58, 1999-2010. [CrossRef]

44. Wang, J.-N.; Kuang, J.-F.; Shan, W.; Chen, J.; Xie, H.; Lu, W.-J.; Chen, J.-W.; Chen, J.-Y. Expression profiles of a banana fruit linker histone $\mathrm{H} 1$ gene MaHIS1 and its interaction with a WRKY transcription factor. Plant Cell Rep. 2012, 31, 1485-1494. [CrossRef]

45. Qi, Y.; Liu, M. Change of endogenous hormone in cultivars of Chinese jujube with different type of embryo abortion. Acta Hortic. Sin. 2004, 6, 800-802.

46. Jiang, Y.; Deyholos, M.K. Comprehensive transcriptional profiling of NaCl-stressed Arabidopsis roots reveals novel classes of responsive genes. BMC Plant Biol. 2006, 6, 25. [CrossRef]

47. Ding, Z.J.; Yan, J.Y.; Li, C.X.; Li, G.X.; Wu, Y.R.; Zheng, S.J. Transcription factor WRKY46 modulates the development of Arabidopsis lateral roots in osmotic/salt stress conditions via regulation of ABA signaling and auxin homeostasis. Plant J. 2015, 84, 56-69. [CrossRef]

48. Chen, L.; Song, Y.; Li, S.; Zhang, L.; Zou, C.; Yu, D. The role of WRKY transcription factors in plant abiotic stresses. BBA-Gene Regul. Mech. 2012, 1819, 120-128. [CrossRef]

49. Chen, H.; Lai, Z.; Shi, J.; Xiao, Y.; Chen, Z.; Xu, X. Roles of Arabidopsis WRKY18, WRKY40 and WRKY60 transcription factors in plant responses to abscisic acid and abiotic stress. BMC Plant Biol. 2010, 10, 281. [CrossRef]

50. Liao, C.J.; Lai, Z.; Lee, S.; Yun, D.J.; Mengiste, T. Arabidopsis HOOKLESS1 regulates responses to pathogens and abscisic acid through interaction with MED18 and acetylation of WRKY33 and ABI5 chromatin. Plant Cell 2016, 28, 1662. [CrossRef]

51. Jiang, Y.; Deyholos, M.K. Functional characterization of Arabidopsis NaCl-inducible WRKY25 and WRKY33 transcription factors in abiotic stresses. Plant Mol. Biol. 2009, 69, 91-105. [CrossRef]

(C) 2019 by the authors. Licensee MDPI, Basel, Switzerland. This article is an open access article distributed under the terms and conditions of the Creative Commons Attribution (CC BY) license (http://creativecommons.org/licenses/by/4.0/). 